\title{
Relativistic self-gravitating Bose-Einstein condensates and cold baryons with a stiff equation of state
}

\author{
Pierre-Henri Chavanis \\ Laboratoire de Physique Théorique, Université Paul Sabatier, 118 route de Narbonne 31062 Toulouse, France
}

\begin{abstract}
Because of their superfluid properties, some compact astrophysical objects such as neutron stars may contain a significant part of their matter in the form of a Bose-Einstein condensate (BEC). We consider a partially-relativistic model of self-gravitating BECs where the relation between the pressure and the rest-mass density is assumed to be quadratic (as in the case of classical BECs) but pressure effects are taken into account in the relation between the energy density and the rest-mass density. At high densities, we get a stiff equation of state similar to the one considered by Zel'dovich (1961) in the context of baryon stars in which the baryons interact through a vector meson field. We determine the maximum mass of general relativistic BEC stars described by this equation of state by using the formalism of Tooper (1965). This maximum mass is slightly larger than the maximum mass obtained by Chavanis and Harko (2012) using a fully-relativistic model. We also consider the possibility that dark matter is made of BECs and apply the partially-relativistic model of BECs to cosmology. In this model, we show that the universe experiences a stiff matter phase, followed by a dust matter phase, and finally by a dark energy phase (equivalent to a cosmological constant). The same evolution is obtained in Zel'dovich (1972) model which assumes that initially, near the cosmological singularity, the universe is filled with cold baryons. Interestingly, the Friedmann equations can be solved analytically in that case and provide a simple generalization of the $\Lambda$ CDM model. We point out, however, the limitations of the partially-relativistic model for BECs and show the need for a fully-relativistic one.

PACS numbers: 04.40.Dg, 67.85.Jk, 95.30.Sf, 95.35.+d, 98.80.-k
\end{abstract}

\section{INTRODUCTION}

Bose-Einstein condensates (BEC) play a major role in condensed matter physics [1]. Recently, it has been suggested that they could play an important role in astrophysics and cosmology also. Indeed, dark matter halos could be quantum objects made of BECs. The wave properties of dark matter may stabilize the system against gravitational collapse providing halo cores instead of cuspy profiles that are predicted by the cold dark matter (CDM) model [2] but not observed [3, 4]. The resulting coherent configuration may be understood as the ground state of some gigantic bosonic atom where the boson particles are condensed in a single macroscopic quantum state $\psi(\mathbf{r})$. In the BEC model, the formation of dark matter structures at small scales is suppressed by quantum mechanics. This property could alleviate the problems of the CDM model such as the cusp problem [3] and the missing satellite problem [5]. At the scale of galaxies, Newtonian gravity can be used so the evolution of the wave function $\psi(\mathbf{r}, t)$ is governed by the GrossPitaevskii-Poisson (GPP) system. The Gross-Pitaevskii (GP) equation [6, 7] is valid at $T=0$ which is relevant for the most compact dwarf halos. Using the Madelung [8] transformation, the GP equation turns out to be equivalent to hydrodynamic (Euler) equations involving an isotropic pressure due to short-range interactions (scattering) and an anisotropic quantum pressure arising from the Heisenberg uncertainty principle. At large scales, quantum effects are negligible and one recovers the classical hydrodynamic equations of the CDM model which are remarkably successful in explaining the large-scale structure of the universe [9]. At small-scales, the pressure arising from the Heisenberg uncertainty principle or from the repulsive scattering of the bosons may stabilize dark matter halos against gravitational collapse and lead to smooth core densities instead of cuspy density profiles in agreement with the observations [3]. Quantum mechanics may therefore be a way to solve the problems of the CDM model.

The possibility that dark matter could be in the form of BECs has a long history (see recent reviews in 10 12]). In some works [13 35], it is assumed that the bosons have no self-interaction. In that case, gravitational collapse is prevented by the Heisenberg uncertainty principle which is equivalent to a quantum pressure. This leads to a mass-radius relation $M R=9.95 \hbar^{2} / G m^{2}$ [14, 32, 36]. In order to account for the mass and size of dwarf dark matter halos, the mass of the bosons must be extremely small, of the order of $m \sim 2.57 \times 10^{-20} \mathrm{eV} / c^{2}$ (see Appendix D of [37]). Ultralight scalar fields like axions may have such small masses (multidimensional string theories predict the existence of bosonic particles down to masses of the order of $\left.m \sim 10^{-33} \mathrm{eV} / c^{2}\right)$. This corresponds to "fuzzy cold dark matter" [19]. In other works [31, 32, 38 51], it is assumed that the bosons have a repulsive self-interaction measured by the scattering length $a_{s}>0$. In that case, gravitational collapse is prevented by the pressure arising from the scattering. In the Thomas-Fermi (TF) approximation which amounts to neglecting the quantum pressure, the resulting structure is equivalent to a polytrope of index $n=1$ with an equation of state $P=2 \pi \hbar^{2} a_{s} \rho^{2} / m^{3}$ [52]. Its radius is given by $R=\pi\left(a_{s} \hbar^{2} / G m^{3}\right)^{1 / 2}$ [31, 40, 42, 43], 
independent on its mass $M$. In order to account for the size of dwarf dark matter halos, the ratio between the mass and the scattering length of the bosons is fixed at $\left(\mathrm{fm} / a_{s}\right)^{1 / 3}\left(m c^{2} / \mathrm{eV}\right)=0.654$ (see Appendix D of [37]). For $a_{s}=10^{6} \mathrm{fm}$, corresponding to the value of the scattering length observed in terrestrial BEC experiments [52], this gives a boson mass $m=65.4 \mathrm{eV} / \mathrm{c}^{2}$ much larger than the mass $m \sim 2.57 \times 10^{-20} \mathrm{eV} / c^{2}$ required in the absence of self-interaction. ${ }^{1}$ This may be more realistic from a particle physics point of view. The general mass-radius relation of self-gravitating BECs at $T=0$ with an arbitrary scattering length $a_{s}$, connecting the non-interacting limit $\left(a_{s}=0\right)$ to the TF limit $\left(G M^{2} m a_{s} / \hbar^{2} \gg 1\right)$, has been determined analytically and numerically in 31, 32. These papers also provide the general density profile of dark matter halos interpreted as self-gravitating BECs at $T=0$ (solitons). The effect of a finite temperature has been considered in $56-$ 63] using different approaches.

Since atoms like ${ }^{7} \mathrm{Li}$ have negative scattering lengths in terrestrial BEC experiments [52], it may be relevant to consider the possibility of self-gravitating BECs with an attractive self-interaction $\left(a_{s}<0\right)$. In that case, there exist a maximum mass $M_{\max }=1.01 \hbar / \sqrt{\left|a_{s}\right| G m}=$ $5.07 M_{P} / \sqrt{|\lambda|}$, where $\lambda=8 \pi a_{s} m c / \hbar$ is the selfinteraction constant and $M_{P}=(\hbar c / G)^{1 / 2}$ is the Planck mass, above which the BEC collapses [31, 32]. In most applications, this mass is extremely small (when $|\lambda| \sim 1$ it is of the order of the Planck mass $M_{P}=2.18 \times 10^{-8} \mathrm{~kg}$ !) so that the collapse of the BEC is very easily realized in the presence of an attractive self-interaction. This may lead to the formation of supermassive black holes at the center of galaxies [12]. On the other hand, when the BEC hypothesis is applied in a cosmological context, an attractive self-interaction can enhance the Jeans instability and accelerate the formation of structures in the universe [64].

Self-gravitating BECs have also been proposed to describe boson stars [36, 65 86]. For these compact objects, we must use general relativity and couple the KleinGordon equation to the Einstein field equations. Initially, the study of boson stars was motivated by the axion field, a pseudo-Nambu-Goldstone boson of the Peccei-Quinn phase transition, that was proposed as a possible solution to the strong CP problem in QCD. In the early works of Kaup [65] and Ruffini \& Bonazzola [36], it was assumed that the bosons have no self-interaction. This leads to

\footnotetext{
1 Actually, using the constraint $4 \pi a_{s}^{2} / m<1.25 \mathrm{~cm}^{2} / \mathrm{g}$ set by the Bullet Cluster [53], implying $\left(a_{s} / \mathrm{fm}\right)^{2}\left(\mathrm{eV} / m c^{2}\right)<1.77 \times 10^{-8}$, one finds the upper bounds $m=1.69 \times 10^{-2} \mathrm{eV} / c^{2}$ (in agreement with the limit $m<1.87 \mathrm{eV} / c^{2}$ obtained from cosmological considerations [54]) and $a_{s}=1.73 \times 10^{-5} \mathrm{fm}$. For a value of the boson mass $m=1.69 \times 10^{-2} \mathrm{eV} / c^{2}$, we have $T \ll T_{c}$ for all the dark matter halos so they can be considered to be at $T=0$ [55]. They are made of a solitonic core surrounded by a halo of scalar radiation.
}

a maximum mass of boson stars equal to $M_{\text {Kaup }}=$ $0.633 M_{P}^{2} / m$. Above that mass no equilibrium configuration exists. In that case, the system collapses into a black hole. This maximum mass is much smaller than the maximum mass $M_{O V}=0.376 M_{P}^{3} / m^{2}$ of fermion stars determined by Oppenheimer and Volkoff [87] in general relativity. They differ by a factor $m / M_{P} \ll 1$. This is because boson stars are stopped from collapsing by Heisenberg's uncertainty principle while, for fermion stars, gravitational collapse is avoided by Pauli's exclusion principle. For $m \sim 1 \mathrm{GeV} / c^{2}$, corresponding to the typical mass of the neutrons, the Kaup mass $M_{\text {Kaup }} \sim 10^{-19} M_{\odot}$ is very small. This corresponds to mini boson stars like axion black holes. The mass of these mini boson stars may be too small to be astrophysically relevant. They could play a role, however, if they exist in the universe in abundance or if the axion mass is extraordinary small leading to macroscopic objects with a mass $M_{\text {Kaup }}$ comparable to the mass of the sun (or even larger) [84]. For example, axionic boson stars could account for the mass of MACHOs (between 0.3 and $0.8 M_{\odot}$ ) if the axions have a mass $m \sim 10^{-10} \mathrm{eV} / c^{2}$ [81]. It has also been proposed that stable boson stars with a boson mass $m \sim 10^{-17} \mathrm{eV} / c^{2}$ could mimic supermassive black holes $\left(M \sim 10^{6} M_{\odot}, R \sim 10^{7} \mathrm{~km}\right)$ that reside at the center of galaxies [82, 85]. On the other hand, Colpi et al. [69] assumed that the bosons have a repulsive self-interaction. In the Thomas-Fermi approximation, this leads to a maximum mass $M_{\max }=0.0612 \sqrt{\lambda} M_{P}^{3} / m^{2}$ which, for $\lambda \sim 1$, is of the order of the maximum mass of fermion stars $M_{O V}=0.376 M_{P}^{3} / \mathrm{m}^{2}$. The self-interaction has the same effect on the bosons as the exclusion principle on the fermions. It plays the role of an interparticle repulsion (for $\lambda>0$ ) that dominates over uncertainty pressure and prevents catastrophic gravitational collapse. Therefore, for $m \sim 1 \mathrm{GeV} / c^{2}$ and $\lambda \sim 1$, we get a maximum mass of the order of the solar mass $M_{\odot}$, similar to the mass of neutron stars, which is much larger than the maximum mass $M_{\text {Kaup }} \sim 10^{-19} M_{\odot}$ obtained in the absence of self-interaction (an interpolation formula giving the maximum mass for any value of the self-interaction constant $\lambda$ is given in Appendix B.5 of [31]). Therefore, a self-interaction can significantly change the physical dimensions of boson stars, making them much more astrophysically interesting. For example, stellar mass boson stars could constitute a part of dark matter [69, 81].

Recently, Chavanis and Harko [86] have proposed that, because of the superfluid properties of the core of neutron stars, the neutrons (fermions) could form Cooper pairs and behave as bosons of mass $2 m_{n}$, where $m_{n}=$ $0.940 \mathrm{Gev} / \mathrm{c}^{2}$ is the mass of the neutrons. Therefore, neutron stars could actually be BEC stars! Since the maximum mass of BEC stars $M_{\max }=0.0612 \sqrt{\lambda} M_{P}^{3} / m^{2}=$ $0.307 \hbar c^{2} \sqrt{a_{s}} /(G m)^{3 / 2}$ depends on the self-interaction constant $\lambda$ (or scattering length $a_{s}$ ), this allows to overcome the (fixed) maximum mass of neutron stars $M_{O V}=$ $0.376 M_{P}^{3} / m^{2}=0.7 M_{\odot}$ determined by Oppenheimer and Volkoff [87] by modeling a neutron star as an ideal 
gas of fermions of mass $m_{n}$ (the corresponding radius is $R=9.36 G M_{O V} / c^{2}=9.6 \mathrm{~km}$ and the corresponding density is $\left.\rho=5 \times 10^{15} \mathrm{~g} / \mathrm{cm}^{3}\right)$. By taking a scattering length of the order of $10-20 \mathrm{fm}$ (hence $\lambda / 8 \pi \sim 95.2-190$ ), we obtain a maximum mass of the order of $2 M_{\odot}$, a central density of the order $1-3 \times 10^{15} \mathrm{~g} / \mathrm{cm}^{3}$, and a radius in the range $10-20 \mathrm{~km}$. This could account for the recently observed neutron stars with masses in the range of $2-2.4 M_{\odot}$ [88 92 much larger than the OppenheimerVolkoff limit 87]. For $M>M_{\max }$, nothing prevents the gravitational collapse of the star which becomes a black hole. On the other hand, for a boson mass of the order of $m \sim 1 \mathrm{MeV} / \mathrm{c}^{2}$ and a self-interaction constant $\lambda \sim 1$, we get $M_{\max } \sim 10^{6} M_{\odot}$ and $R_{\min } \sim 10^{7} \mathrm{~km}$. These parameters are reminiscent of supermassive black holes in active galactic nuclei, so that stable self-interacting boson stars with $m \sim 1 \mathrm{MeV} / \mathrm{c}^{2}$ could be an alternative to black holes at the center of galaxies [80].

Self-gravitating BECs may also find applications in the physics of black holes [12]. It has been proposed recently that microscopic quantum black holes could be BECs of gravitons stuck at a critical point 93, 94. These results can be understood easily in terms of the Kaup mass and Kaup radius [12]. Therefore, self-gravitating BECs can have many applications in astrophysics, cosmology and black hole physics with promising perspectives.

In this paper, we come back to certain approximations that have been made in the study of self-gravitating BECs and discuss them in more detail.

In their study of general relativistic BEC stars, Chavanis and Harko [86] first presented qualitative arguments giving the fundamental scalings of the maximum mass $M_{*} \sim \hbar c^{2} \sqrt{a_{s}} /(G m)^{3 / 2}$, minimum radius $R_{*} \sim\left(a_{s} \hbar^{2} / G m^{3}\right)^{1 / 2}$, and maximum density $\rho_{*} \sim$ $m^{3} c^{2} / 2 \pi a_{s} \hbar^{2}$ of BEC stars. Then, they developed two models in order to obtain the numerical values of the prefactors. They first developed a semi-relativistic model in which gravity is treated by general relativity using the Tolman-Oppenheimer-Volkoff (TOV) equation but the relation between the pressure and the energy density is given by the quadratic equation of state $P=2 \pi \hbar^{2} a_{s} \epsilon^{2} / m^{3} c^{4}$ obtained from the classical GrossPitaevskii equation after identifying the energy density with the rest-mass density $\left(\epsilon=\rho c^{2}\right)$. This is a particular case of a polytropic equation of state, corresponding to an index $n=1$, studied by Tooper [95] in general relativity. This semi-relativistic model leads to a maximum mass $M_{\max }=0.5001 \hbar c^{2} \sqrt{a_{s}} /(G m)^{3 / 2}$. This treatment is approximate first because the energy density is not always dominated by the rest-mass density and also because the relation between the pressure and the restmass density is altered by relativistic effects. Chavanis and Harko [86] also developed a fully-relativistic model in which the relation between the pressure and the energy density is obtained from the Klein-Gordon equation [69]. In the dense core, the equation of state reduces to $P \sim \epsilon / 3$ which is similar to the equation of state of the radiation or to the equation of state that pre- vails in the core of neutron stars modeled as an ideal gas of fermions at $T=0$. In the envelope, we recover the equation of state $P=2 \pi \hbar^{2} a_{s} \epsilon^{2} / m^{3} c^{4}$ of a classical BEC. This fully-relativistic model leads to a maximum mass $M_{\max }=0.307 \hbar c^{2} \sqrt{a_{s}} /(G m)^{3 / 2}$. This is the correct value of the maximum mass of BEC stars. In this paper, we shall compare these results with a partiallyrelativistic model of self-gravitating BECs where the relation between the pressure and the rest-mass density is assumed to be given by $P=2 \pi \hbar^{2} a_{s} \rho^{2} / m^{3}$ (as for a classical BEC) but pressure effects are taken into account in the relation between the energy density and the restmass density $\left(\epsilon=\rho c^{2}+P\right)$. This is a particular case of an equation of state studied by Tooper [96] in general relativity. In the dense core, the equation of state reduces to $P \sim \epsilon$. This is a stiff equation of state for which the velocity of sound $c_{s}=\sqrt{P^{\prime}(\epsilon)} c$ is equal to the velocity of light $\left(c_{s}=c\right)$. This type of equation of state was introduced by Zel'dovich [97] in the context of baryon stars in which the baryons interact through a vector meson field. In the envelope, we recover the equation of state $P=2 \pi \hbar^{2} a_{s} \epsilon^{2} / m^{3} c^{4}$ of a classical BEC. This partially-relativistic model leads to a maximum mass $M_{\max }=0.4104 \hbar c^{2} \sqrt{a_{s}} /(G m)^{3 / 2}$ intermediate between the two models considered by Chavanis and Harko [86]. This treatment is, however, approximate because the relation between the pressure and the rest-mass density is altered by relativistic effects.

Self-gravitating BECs have also been considered in cosmology. Harko [98] and Chavanis [64] independently developed cosmological models in which dark matter is made of BECs. They solved the Friedmann equations by assuming that the equation of state relating the pressure to the energy density is given by $P=2 \pi \hbar^{2} a_{s} \epsilon^{2} / m^{3} c^{4}$, as for Newtonian BECs. However, this equation of state is not valid when the BEC is strongly relativistic. Therefore, their approach gives wrong results in the very early universe where relativistic effects are important. A fullyrelativistic model should use the equation of state derived from the Klein-Gordon equation [69]. This will be considered in a future work. As an intermediate step, we consider here a partially-relativistic model in which the relation between the pressure and the rest-mass density is assumed to be given by $P=2 \pi \hbar^{2} a_{s} \rho^{2} / \mathrm{m}^{3}$ (as for a classical BEC) but pressure effects are taken into account in the relation between the energy density and the rest-mass density $\left(\epsilon=\rho c^{2}+P\right)$. This leads to a cosmological model where the universe experiences a stiff matter phase, followed by a dust matter phase, and finally by a dark energy phase (equivalent to a cosmological constant). The same evolution is obtained in Zel'dovich [99] model which assumes that initially, near the cosmological singularity, the universe is filled with cold baryons. Interestingly, the Friedmann equations can be solved analytically in that case and provide a simple generalization of the $\Lambda \mathrm{CDM}$ model. We point out, however, the limitations of this partially-relativistic model for BECs and the need for a fully-relativistic one. Although our relativistic 
treatment is approximate for BECs, it is exact for the type of particles considered by Zel'dovich [97, 99].

The paper is organized as follows. In Sec. II we recall the basic equations describing Newtonian self-gravitating BECs at $T=0$. We also recall the qualitative arguments of Chavanis and Harko [86] giving the scaling of the maximum mass, minimum radius, and maximum density of relativistic self-gravitating BECs. In Sec. III we determine the maximum mass of general relativistic BECs using a partially-relativistic model and compare the result with the ones obtained by Chavanis and Harko [86] using a semi-relativistic model and a fully-relativistic model. We also discuss the analogies and the differences between models that treat neutron stars as fermion stars or as BEC stars. We finally point out the analogy between BEC stars described by a stiff equation of state and the concept of baryon stars introduced by Zel'dovich [97]. In Sec. IV] we develop a cosmological model in which dark matter is made of BECs with a stiff equation of state. We point out the analogy with the model of Zel'dovich [99] that assumes that the primordial universe is filled with cold baryons. We provide analytical solutions of the Friedmann equations exhibiting a stiff matter era. We also discuss the effect of the BEC equation of state on the evolution of the universe.

\section{SELF-GRAVITATING BOSE-EINSTEIN CONDENSATES}

\section{A. The Gross-Pitaevskii-Poisson system}

At $T=0$, in the Newtonian regime, a self-gravitating $\mathrm{BEC}$ with short-range interactions is described by the Gross-Pitaevskii-Poisson system

$$
\begin{gathered}
i \hbar \frac{\partial \psi}{\partial t}=-\frac{\hbar^{2}}{2 m} \Delta \psi+m \Phi \psi+\frac{4 \pi a_{s} \hbar^{2} N}{m}|\psi|^{2} \psi, \\
\Delta \Phi=4 \pi G N m|\psi|^{2},
\end{gathered}
$$

where $\rho(\mathbf{r}, t)=N m|\psi|^{2}$ is the mass density ( $N$ is the number of bosons and $m$ is their mass), $\psi(\mathbf{r}, t)$ is the wave function, $\Phi(\mathbf{r}, t)$ is the gravitational potential, and $a_{s}$ is the s-scattering length of the bosons. These equations are valid in a mean field approximation which is known to be exact for systems with long-range interactions (such as self-gravitating systems) when $N \rightarrow+\infty$.

Using the Madelung [8] transformation

$$
\psi=\sqrt{\frac{\rho}{N m}} e^{i S / \hbar}, \quad \mathbf{u}=\frac{1}{m} \nabla S,
$$

where $S(\mathbf{r}, t)$ is an action and $\mathbf{u}(\mathbf{r}, t)$ is an irrotational velocity field, we can rewrite the GPP system (11)-(2) in the form of hydrodynamic equations

$$
\frac{\partial \rho}{\partial t}+\nabla \cdot(\rho \mathbf{u})=0
$$

$$
\begin{gathered}
\frac{\partial \mathbf{u}}{\partial t}+(\mathbf{u} \cdot \nabla) \mathbf{u}=-\frac{1}{\rho} \nabla P-\nabla \Phi-\frac{1}{m} \nabla Q, \\
\Delta \Phi=4 \pi G \rho,
\end{gathered}
$$

where

$$
Q=-\frac{\hbar^{2}}{2 m} \frac{\Delta \sqrt{\rho}}{\sqrt{\rho}}
$$

is the quantum potential and

$$
P=\frac{2 \pi a_{s} \hbar^{2}}{m^{3}} \rho^{2}
$$

is the pressure arising from the short-range interaction. It corresponds to a polytropic equation of state

$$
P=K \rho^{\gamma}, \quad \gamma=1+\frac{1}{n}
$$

with a polytropic index $n=1$ (i.e. $\gamma=2$ ) and a polytropic constant

$$
K=\frac{2 \pi \hbar^{2} a_{s}}{m^{3}} .
$$

Eqs. (41)-(7) form the quantum barotropic Euler-Poisson system.

The condition of hydrostatic equilibrium $\left(\partial_{t}=0\right.$ and $\mathbf{u}=\mathbf{0}$ ) writes

$$
\nabla P+\rho \nabla \Phi+\frac{\rho}{m} \nabla Q=\mathbf{0}
$$

It expresses the balance between the gravitational attraction and the repulsion due to the scattering and to the quantum pressure. Combining this equation with the Poisson equation (6) and using Eqs. (7) and (8), we get

$$
\frac{4 \pi a_{s} \hbar^{2}}{m^{3}} \Delta \rho+4 \pi G \rho-\frac{\hbar^{2}}{2 m^{2}} \Delta\left(\frac{\Delta \sqrt{\rho}}{\sqrt{\rho}}\right)=0 .
$$

This differential equation determines the density profile of a self-gravitating BEC. It is equivalent to the stationary solution (soliton) of the GPP system [12, 31]. It has been solved analytically (approximately) and numerically (exactly) in Refs. 31] and [32] for arbitrary values of the scattering length $a_{s}$ and of the boson mass $m$.

\section{B. The Thomas-Fermi approximation}

In the TF approximation valid when $G M^{2} m a_{s} / \hbar^{2} \gg$ 1 , we can neglect the contribution of the quantum potential. In that case, the condition of hydrostatic equilibrium reduces to the usual form

$$
\nabla P+\rho \nabla \Phi=\mathbf{0}
$$

and the differential equation (12) becomes

$$
\frac{4 \pi a_{s} \hbar^{2}}{m^{3}} \Delta \rho+4 \pi G \rho=0
$$


Writing $\rho=\rho_{0} \theta$ and $r=\left(a_{s} \hbar^{2} / G m^{3}\right)^{1 / 2} \xi$, where $\rho_{0}$ is the central density, and considering a spherically symmetric system, this equation can be put in the form of the LaneEmden equation

$$
\begin{gathered}
\frac{1}{\xi^{2}} \frac{d}{d \xi}\left(\xi^{2} \frac{d \theta}{d \xi}\right)=-\theta, \\
\theta(0)=1, \quad \theta^{\prime}(0)=0,
\end{gathered}
$$

for a polytrope of index $n=1$ [100]. It has the analytical solution

$$
\theta(\xi)=\frac{\sin \xi}{\xi}
$$

The radius of the configuration is defined by the condition $\theta\left(\xi_{1}\right)=0$, giving $\xi_{1}=\pi$. Therefore the radius $R$ of the self-gravitating $\mathrm{BEC}$ is given by

$$
R=\pi \sqrt{\frac{a_{s} \hbar^{2}}{G m^{3}}} .
$$

It is independent on the central density and on the mass of the system, and depends only on the physical characteristics of the condensate (the mass $m$ and the scattering length $a_{s}$ of the bosons). Actually, it is fixed by the ratio $a_{s} / m^{3}$.

The mass of a self-gravitating BEC star with a quartic non-linearity is given as a function of the central density and of the coherent scattering length $a_{s}$ by

$$
M=4 \pi\left(\frac{a_{s} \hbar^{2}}{G m^{3}}\right)^{3 / 2} \rho_{0} \xi_{1}^{2}\left|\theta^{\prime}\left(\xi_{1}\right)\right|,
$$

yielding

$$
M=4 \pi^{2}\left(\frac{a_{s} \hbar^{2}}{G m^{3}}\right)^{3 / 2} \rho_{0},
$$

where we have used $\left|\theta^{\prime}\left(\xi_{1}\right)\right|=1 / \pi$. Using Eq. (18), it can be expressed in terms of the radius and central density by

$$
M=\frac{4}{\pi} \rho_{0} R^{3},
$$

which shows that the mean density of the configuration $\bar{\rho}=3 M / 4 \pi R^{3}$ is related to the central density by the relation $\bar{\rho}=3 \rho_{0} / \pi^{2}$. Other quantities of interest such as the energy and the moment of inertia are derived in [31].

\section{Maximum mass of relativistic BEC stars: qualitative treatment and fundamental scalings}

The Newtonian treatment of self-gravitating BECs is appropriate to describe dark matter halos. However, general relativistic effects may be important in the case of
BEC stars describing compact objects such as neutron stars or dark matter stars [12].

The radius of a Newtonian BEC star is given by Eq. (18). In the Newtonian treatment, there is no limit on the mass of the BEC. However, the Newtonian treatment breaks down when the radius of the star approaches the Schwarzschild radius $R_{S}=2 G M / c^{2}$. Equating the two radii, namely writing $M=R c^{2} / 2 G$ with $R$ given by Eq. (18), and ignoring the prefactors that are necessarily inexact, we obtain the scaling of the maximum mass and of the minimum radius of a relativistic BEC star [86]:

$$
\begin{gathered}
M_{*}=\frac{\hbar c^{2} \sqrt{a_{s}}}{(G m)^{3 / 2}}=1.420 \kappa M_{\odot}, \\
R_{*}=\frac{G M_{*}}{c^{2}}=\left(\frac{a_{s} \hbar^{2}}{G m^{3}}\right)^{1 / 2}=2.106 \kappa \mathrm{km},
\end{gathered}
$$

where we have introduced the dimensionless parameter

$$
\kappa=\left(\frac{a_{s}}{1 \mathrm{fm}}\right)^{1 / 2}\left(\frac{m}{2 m_{n}}\right)^{-3 / 2} .
$$

From Eqs. (22) and (23), we obtain the scaling of the maximum central density

$$
\rho_{*}=\frac{m^{3} c^{2}}{2 \pi a_{s} \hbar^{2}}=4.846 \times 10^{16} \kappa^{-2} \mathrm{~g} / \mathrm{cm}^{3},
$$

where the factor $2 \pi$ has been introduced for future convenience.

We note that the expression of the scaled radius $R_{*}$ is the same as in the Newtonian regime (it is independent on $c$ ) while the scaling of the mass and of the density are determined by relativistic effects.

\section{GENERAL RELATIVISTIC BOSE-EINSTEIN CONDENSATE STARS}

For a correct determination of the maximum mass of BEC stars, we cannot ignore the effects induced by the space-time curvature, and a general relativistic treatment is necessary.

\section{A. The Tolman-Oppenheimer-Volkoff equation}

For a static spherically symmetric star, the interior line element is given by

$$
d s^{2}=e^{\nu(r)} c^{2} d t^{2}-e^{\lambda(r)} d r^{2}-r^{2}\left(d \theta^{2}+\sin ^{2} \theta d \phi^{2}\right) .
$$

The equations describing a general relativistic compact star are the mass continuity equation and the TolmanOppenheimer-Volkoff (TOV) equation. They write [101]:

$$
\frac{d M}{d r}=4 \pi \frac{\epsilon}{c^{2}} r^{2}
$$




$$
\frac{d P}{d r}=-\frac{G(\epsilon+P)\left[4 \pi P r^{3} / c^{2}+M(r)\right]}{r^{2} c^{2}\left[1-2 G M(r) / c^{2} r\right]},
$$

where $\epsilon$ is the energy density and $M(r)$ is the total mass interior to $r$. The mass of the star is $M=M(R)$ where $R$ is its radius. These equations extend the classical condition of hydrostatic equilibrium for a self-gravitating gas to the context of general relativity. The system of equations (27)-(28) must be closed by choosing the equation of state $P=P(\epsilon)$ for the thermodynamic pressure. At the center of the star, the mass must satisfy the boundary condition $M(0)=0$. For the thermodynamic pressure $P$, we assume that it vanishes on the surface: $P(R)=0$.

The exterior of the star is characterized by the Schwarzschild metric, describing the vacuum $(P=\epsilon=0)$ outside the star, and given by [101]:

$$
\left(e^{\nu}\right)^{\mathrm{ext}}=\left(e^{-\lambda}\right)^{\mathrm{ext}}=1-\frac{2 G M}{c^{2} r}, \quad r \geq R .
$$

The interior solution must match with the exterior solution on the vacuum boundary of the star. by

The components of the metric tensor are determined

$$
\begin{gathered}
e^{-\lambda(r)}=1-\frac{2 G M(r)}{r c^{2}} \\
\frac{d P}{d r}+\frac{P+\epsilon}{2} \frac{d \nu}{d r}=0, \quad e^{\nu(R)}=1-\frac{2 G M}{R c^{2}} .
\end{gathered}
$$

The boundary condition on $e^{\nu}$ has been chosen so that this component is continuous with the exterior solution at $r=R$.

\section{B. Maximum mass of relativistic BEC stars with short-range interactions: partially-relativistic treatment}

We consider a partially-relativistic model (see Appendix A3) in which the BEC star is described in general relativity by the equation of state

$$
P=K \rho^{2}, \quad \epsilon=\rho c^{2}+P,
$$

where $K$ is given by Eq. (10). Here, $\epsilon$ is the energy density and $\rho$ is the rest-mass density. It is related to the number density $n$ by $\rho=m n$. The pressure can be expressed as a function of the energy density as (see Appendix A3):

$$
P=\frac{c^{4}}{4 K}\left(\sqrt{1+\frac{4 K \epsilon}{c^{4}}}-1\right)^{2} .
$$

In the non-relativistic regime $(\epsilon \rightarrow 0)$, we recover the classical equation of state of a BEC star $P \sim K \epsilon^{2} / c^{4} \sim$ $K \rho^{2}$. In the ultra-relativistic regime $(\epsilon \rightarrow+\infty)$, we obtain a stiff equation of state $P \sim \epsilon$ in which the velocity of sound is equal to the velocity of light. A stiff equation of state was first introduced by Zel'dovich [97] in the context of baryon stars in which the baryons interact through a vector meson field (see Sec. IIIE). We know that a linear equation of state $P=q \epsilon$ leads to a mass-central density relation that presents damped oscillations, and to a mass-radius relation that has a spiral structure 102, 103]. Therefore, the series of equilibria of BEC stars described by the equation of state (33) will exhibit this behavior. This is similar to the series of equilibria of neutron stars modeled as a gas of relativistic fermions that have a linear equation of state $P \sim \epsilon / 3$ for $\epsilon \rightarrow+\infty$ (see Sec. [IID) 87, 104, 105]. This is also similar to the series of equilibria of isothermal spheres described by a linear equation of state $P=\rho k_{B} T / m$ in Newtonian gravity [106].

The equation of state (32) is a particular case, corresponding to a polytropic index $n=1$, of the class of equations of state studied by Tooper [96] in general relativity. We shall use his formalism and notations. Therefore, we set

$$
\begin{gathered}
\rho=\rho_{0} \theta, \quad r=\frac{\xi}{A}, \quad \sigma=\frac{K \rho_{0}}{c^{2}}, \\
M(r)=\frac{4 \pi \rho_{0}}{A^{3}} v(\xi), \quad A=\left(\frac{2 \pi G}{K}\right)^{1 / 2},
\end{gathered}
$$

where $\rho_{0}$ is the central rest-mass density and $\sigma$ is the relativity parameter. In terms of these variables, the TOV equation and the mass continuity equation become

$$
\begin{gathered}
\frac{d \theta}{d \xi}=-\frac{(1+2 \sigma \theta)\left(v+\sigma \xi^{3} \theta^{2}\right)}{\xi^{2}(1-4 \sigma v / \xi)}, \\
\frac{d v}{d \xi}=\theta \xi^{2}(1+\sigma \theta) .
\end{gathered}
$$

For a given value of the relativity parameter $\sigma$, they have to be solved with the initial condition $\theta(0)=1$ and $v(0)=0$. Since $v \sim \xi^{3}$ as $\xi \rightarrow 0$, it is clear that $\theta^{\prime}(0)=0$. On the other hand, the density vanishes at the first zero $\xi_{1}$ of $\theta: \theta\left(\xi_{1}\right)=0$. This determines the boundary of the star. In the non-relativistic limit $\sigma \rightarrow 0$, the system of equations (36)-(37) reduces to the Lane-Emden equation (15) with $n=1$.

From the foregoing relations, we find that the radius, the mass and the central density of the configuration are given by

$$
R=\xi_{1} R_{*}, \quad M=2 \sigma v\left(\xi_{1}\right) M_{*}, \quad \rho_{0}=\sigma \rho_{*},
$$

where

$$
R_{*}=\left(\frac{K}{2 \pi G}\right)^{1 / 2}, \quad M_{*}=\left(\frac{K c^{4}}{2 \pi G^{3}}\right)^{1 / 2}, \quad \rho_{*}=\frac{c^{2}}{K} .
$$

For the value of $K$ given by Eq. (10), one can check that the fundamental scaling parameters $R_{*}, M_{*}$ and $\rho_{*}$ are 
given by Eqs. (22)- (25). By varying $\sigma$ from 0 to $+\infty$, we obtain the series of equilibria in the form $M\left(\rho_{0}\right)$ and $R\left(\rho_{0}\right)$. We can then plot the mass-radius relation $M(R)$ parameterized by $\rho_{0}$.

Using the Poincaré theorem [107] (see also [108, 109]), one can show [102, 103] that the series of equilibria becomes unstable after the first mass peak and that a new mode of instability appears at each turning point of mass in the series of equilibria (see [110 for an alternative derivation of these results based on the equation of pulsations). These results of dynamical stability for general relativistic stars are similar to results of dynamical and thermodynamical stability for Newtonian self-gravitating systems [106, 111].

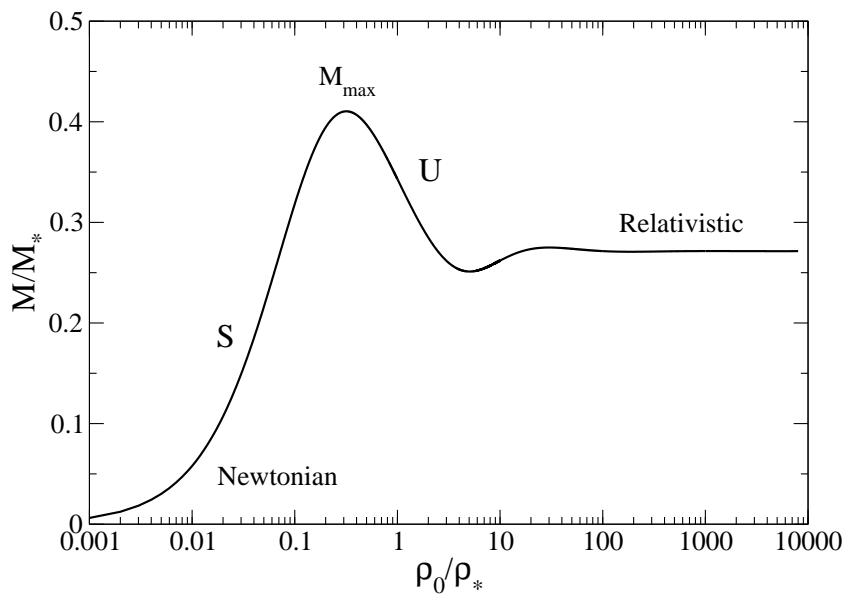

FIG. 1: Dimensionless mass-central density relation of a relativistic BEC with short-range interactions modeled by the equation of state (32). There exist a maximum mass $M_{\max } / M_{*}=0.4104$ at which the series of equilibria becomes dynamically unstable. The velocity of sound is always smaller than the velocity of light. We note that the mass-central density relation presents damped oscillations at high densities similarly to neutron stars described by a fermionic equation of state [87, 102 105].

The series of equilibria corresponding to the equation of state (32) is represented in Figs. 113. These figures respectively give the mass-central density relation, the radius-central density relation, and the mass-radius relation. Some density profiles are plotted in Fig. 4. The series of equilibria is parameterized by the relativity parameter $\sigma$ going from $\sigma=0$ (non-relativistic) to $\sigma \rightarrow+\infty$ (ultra-relativistic). The configurations are stable for $\sigma \leq \sigma_{c}$ and unstable for $\sigma \geq \sigma_{c}$ where

$$
\sigma_{c}=0.318
$$

corresponds to the first turning point of mass: $M^{\prime}\left(\sigma_{c}\right)=$ 0 . The values of $\xi_{1}$ and $v\left(\xi_{1}\right)$ at this point are

$$
\xi_{1}=1.914, \quad v\left(\xi_{1}\right)=0.6453 .
$$

The corresponding values of radius, mass and central

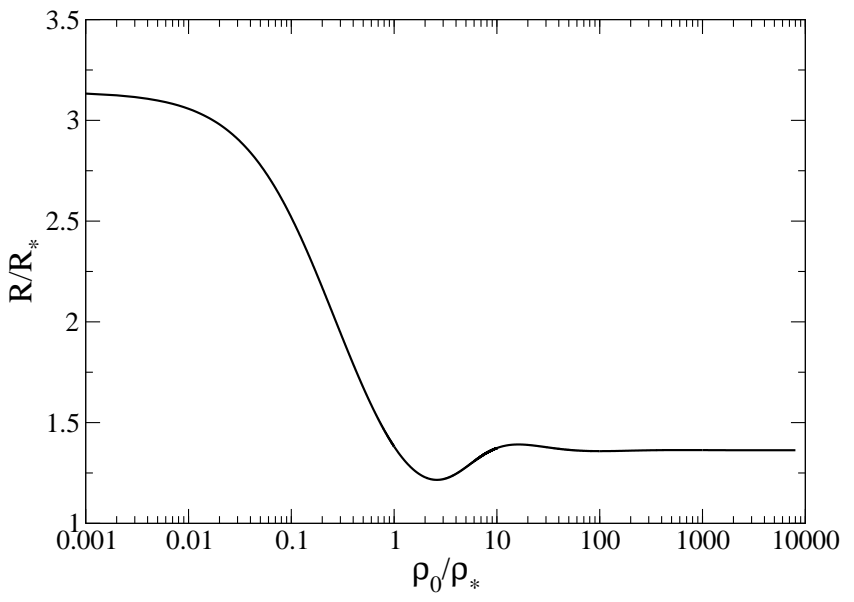

FIG. 2: Dimensionless radius-central density relation of a relativistic BEC with short-range interactions modeled by the equation of state (32).

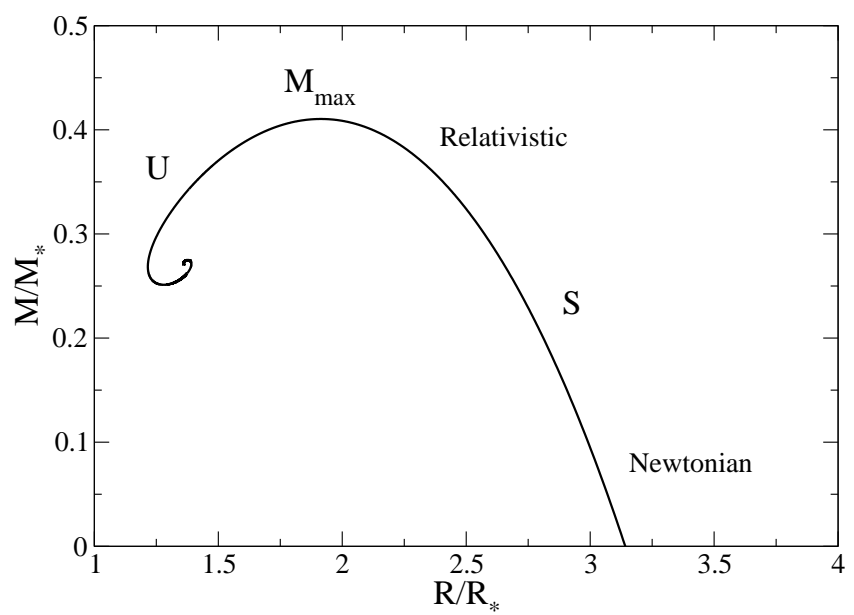

FIG. 3: Dimensionless mass-radius relation of a relativistic BEC with short-range interactions modeled by the equation of state (32). The series of equilibria is parameterized by the relativity parameter $\sigma$. The mass-radius relation presents a snail-like structure (spiral) at high densities similarly to neutron stars described by a fermionic equation of state 87 , 102 105]. There exist a maximum mass $M_{\max } / M_{*}=0.4104$ and a minimum radius $R_{\min } / R_{*}=1.914$ corresponding to a maximum central density $\left(\rho_{0}\right)_{\max }=0.318 \rho_{*}$. There also exist a maximum radius $R_{\max } / R_{*}=\pi$ corresponding to the Newtonian limit $\sigma \rightarrow 0$.

density are

$$
\begin{gathered}
R_{\min }=1.914\left(\frac{a_{s} \hbar^{2}}{G m^{3}}\right)^{1 / 2}=4.03 \kappa \mathrm{km}, \\
M_{\max }=0.4104 \frac{\hbar c^{2} \sqrt{a_{s}}}{(G m)^{3 / 2}}=0.583 \kappa M_{\odot}, \\
\left(\rho_{0}\right)_{\max }=0.318 \frac{m^{3} c^{2}}{2 \pi a_{s} \hbar^{2}}=1.54 \times 10^{16} \kappa^{-2} \mathrm{~g} / \mathrm{cm}^{3},
\end{gathered}
$$




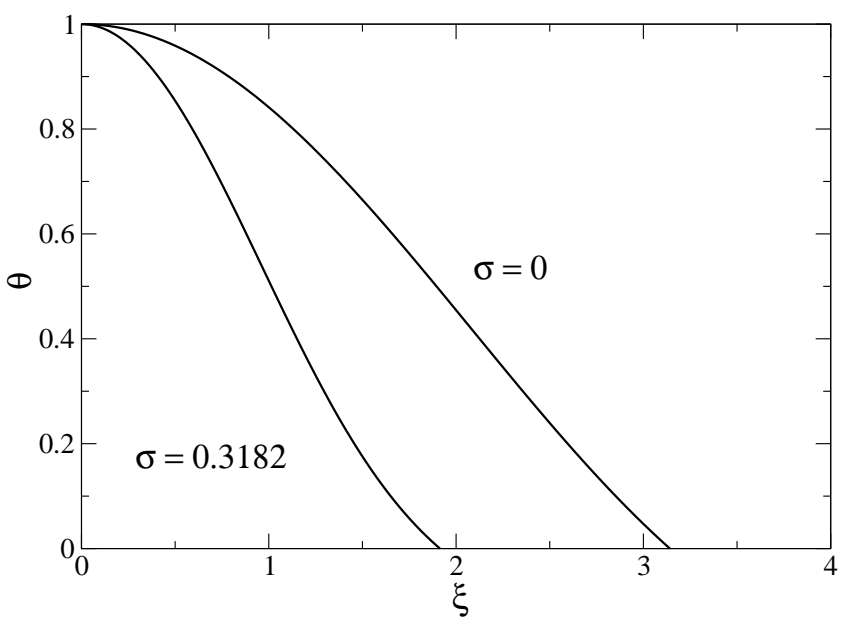

FIG. 4: Dimensionless density profiles corresponding to $\sigma=0$ (Newtonian) and $\sigma=\sigma_{c}=0.318$ (maximum mass).

respectively. They define the minimum radius, the maximum mass, and the maximum rest-mass density of the stable configurations.

The energy density is related to the rest-mass density by Eq. (32) which can be rewritten as

$$
\epsilon / c^{2}=\rho\left(1+\rho \frac{2 \pi \hbar^{2} a_{s}}{m^{3} c^{2}}\right) .
$$

Using Eqs. (44) and (45), the maximum energy density is

$$
\left(\epsilon_{0}\right)_{\max } / c^{2}=0.419 \frac{m^{3} c^{2}}{2 \pi a_{s} \hbar^{2}}=2.03 \times 10^{16} \kappa^{-2} \mathrm{~g} / \mathrm{cm}^{3} .
$$

We note that the radius of a relativistic BEC star is necessarily smaller than

$$
R_{\max }=\pi \sqrt{\frac{\hbar^{2} a_{s}}{G m^{3}}}=6.61 \kappa \mathrm{km},
$$

corresponding to the Newtonian limit $(\sigma \rightarrow 0)$. The Newtonian approximation is valid for small masses $M \ll$ $M_{\max }$. The radius decreases as $M$ increases until the maximum mass and the minimum radius are reached. When $M>M_{\max }$, there is no equilibrium state and the BEC star is expected to collapse and form a black hole. When $M<M_{\max }$, there exist stable equilibrium states with $R_{\min }<R<R_{\max }$ that correspond to BEC stars for which gravitational collapse is prevented by quantum mechanics (the self-interaction of the bosons). We note that the radius of the BEC star is very much constrained as it lies in the range $4.03 \kappa \leq R(\mathrm{~km}) \leq 6.61 \kappa$.

A quantity of physical interest is the mass-radius ratio

$$
\frac{2 G M}{R c^{2}}=\frac{4 \sigma v\left(\xi_{1}\right)}{\xi_{1}}
$$

At the critical point, the value of the mass-radius ratio is 0.429 . We check that it is smaller than the Buchdahl maximum bound $2 G M / R c^{2}=8 / 9=0.888$ corresponding to constant density stars [112].

\section{Comparison between the different models}

The values of the maximum mass, minimum radius, and maximum central energy density of general relativistic BEC stars can be written as

$$
\begin{gathered}
R_{\min }=A_{1}\left(\frac{a_{s} \hbar^{2}}{G m^{3}}\right)^{1 / 2}=A_{1}^{\prime} \kappa \mathrm{km}, \\
M_{\max }=A_{2} \frac{\hbar c^{2} \sqrt{a_{s}}}{(G m)^{3 / 2}}=A_{2}^{\prime} \kappa M_{\odot} \\
\left(\epsilon_{0}\right)_{\max } / c^{2}=A_{3} \frac{m^{3} c^{2}}{2 \pi a_{s} \hbar^{2}}=A_{3}^{\prime} \times 10^{16} \kappa^{-2} \mathrm{~g} / \mathrm{cm}^{3},
\end{gathered}
$$

where $\kappa$ is defined by Eq. (24). These scalings are fundamental for BEC stars [6]. However, the values of the prefactors depend on the relativistic model.

The best model is the one based on the equation of state A15 considered in Sec. VI.C. of Chavanis and Harko 86 because this equation of state can be derived from the relativistic Klein-Gordon equation [69]. Therefore, this model is fully-relativistic, both regarding the equation of state and the treatment of gravity. In that model, the prefactors are $A_{1}=1.923, A_{1}^{\prime}=4.047$, $A_{2}=0.307, A_{2}^{\prime}=0.436, A_{3}=0.398$, and $A_{3}^{\prime}=1.929$. They can be considered as being the exact prefactors for relativistic BEC stars.

The model based on the equation of state (A29) considered in Sec. VI.B. of Chavanis \& Harko [86] is very approximate because it is based on an equation of state $P=2 \pi \hbar^{2} a_{s} \rho^{2} / m^{3}$ derived from the classical GP equation and it furthermore assumes that the energy density is dominated by the rest-mass density so that $\epsilon=\rho c^{2}$. Therefore, this model is semi-relativistic because the equation of state is classical while gravity is treated in the framework of general relativity. In that model, the prefactors are $A_{1}=1.888, A_{1}^{\prime}=3.974, A_{2}=0.5001$, $A_{2}^{\prime}=0.710, A_{3}=0.42$, and $A_{3}^{\prime}=2.035$.

The model based on the equation of state (A25) is intermediate between the two previous models. It is based on an equation of state $P=2 \pi \hbar^{2} a_{s} \rho^{2} / \mathrm{m}^{3}$ derived from the classical GP equation but it takes into account the difference between the energy density and the rest-mass density due to pressure effects: $\epsilon=\rho c^{2}+P$ [96]. Therefore, this model is partially-relativistic. In that model, the prefactors are $A_{1}=1.914, A_{1}^{\prime}=4.03, A_{2}=0.4104$, $A_{2}^{\prime}=0.583, A_{3}=0.419$, and $A_{3}^{\prime}=2.03$.

The mass-radius relation of general relativistic BEC stars at $T=0$ corresponding to these different models is plotted in Fig. 5 We note that the values of the prefactors do not differ much from one model to the other. The maximum mass varies between $\sim 0.3 M_{*}$ and $\sim 0.5 M_{*}$ while the minimum radius and the maximum energy density almost do not change.

As discussed specifically in Sec. IIID general relativistic BEC stars may describe neutron stars with a superfluid core. This is why we have normalized the mass 


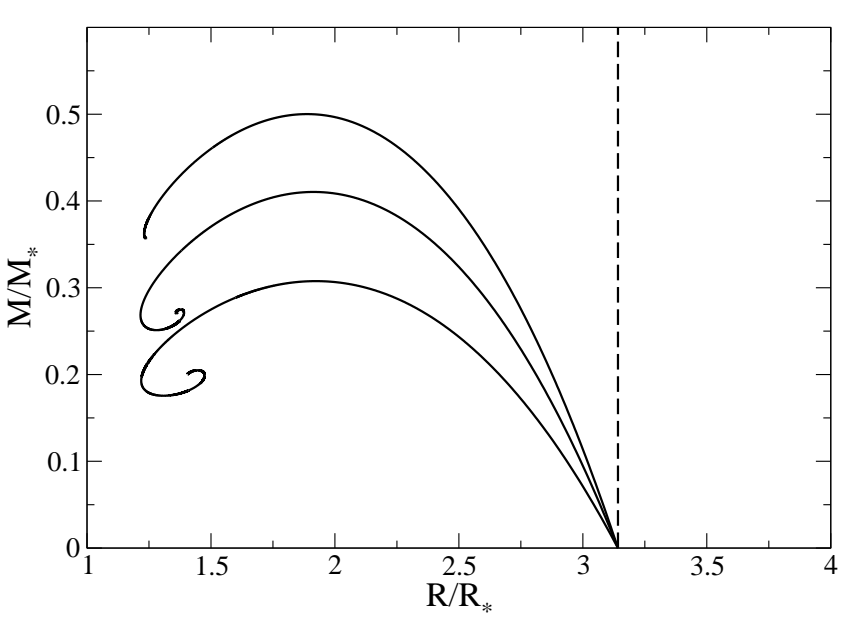

FIG. 5: Comparison between the mass-radius relations corresponding to the fully-relativistic model (lower curve), to the partially-relativistic model (intermediate curve), and to the semi-relativistic model (upper curve). The vertical dashed line corresponds to the Newtonian limit.

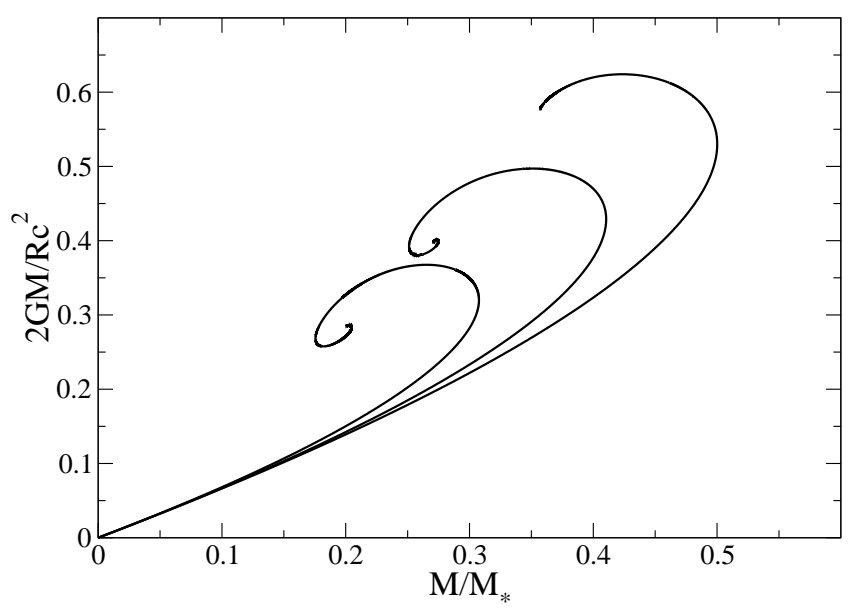

FIG. 6: Mass-radius ratio of general relativistic BEC stars corresponding to the fully-relativistic model (left curve), to the partially-relativistic model (middle curve), and to the semi-relativistic model (right curve). The Buchdahl maximum bound $2 G M / R c^{2}=8 / 9=0.888$ is much higher.

of the bosons by $2 m_{n}$ (Cooper pair) in Eq. (24). However, general relativistic BEC stars may describe other compact objects such as boson stars or dark matter stars [12]. In this respect, it may be convenient to write the maximum mass, the minimum radius, and the maximum
TABLE I: Observational values of the mass and radius of neutron stars.

\begin{tabular}{llll}
\hline Ref. & $M / M_{\odot}$ & $R / \mathrm{km}$ & $2 G M / R c^{2}$ \\
\hline$[113]$ & 1.3 & 8 & 0.479 \\
{$[113]$} & 1.95 & 12 & 0.479 \\
{$[114]$} & 2.1 & 12.5 & 0.4956 \\
{$[115]$} & 1.58 & 9.11 & 0.512 \\
{$[116]$} & 1.9 & 15.2 & 0.369 \\
\hline
\end{tabular}

central density as ${ }^{2}$

$$
\begin{gathered}
\frac{M_{\max }}{M_{\odot}}=A\left(\frac{a}{\mathrm{fm}}\right)^{1 / 2}\left(\frac{\mathrm{GeV} / c^{2}}{m}\right)^{3 / 2}, \\
\frac{R_{\min }}{\mathrm{km}}=B \frac{M_{\max }}{M_{\odot}}, \\
\frac{\left(\epsilon_{0}\right)_{\max } / c^{2}}{10^{16} \mathrm{~g} / \mathrm{cm}^{3}}=C\left(\frac{M_{\odot}}{M_{\max }}\right)^{2} .
\end{gathered}
$$

For the fully-relativistic model, $A=1.12, B=9.27$, and $C=0.364$. For the semi-relativistic model, $A=1.83$, $B=5.59$, and $C=1.02$. For the partially-relativistic model, $A=1.50, B=6.91$, and $C=0.689$. The maximum radius $R_{\max }$ of the star, corresponding to the radius of a Newtonian BEC given by Eq. (47), can be written as

$$
\frac{R_{\max }}{\mathrm{km}}=17.0\left(\frac{a}{\mathrm{fm}}\right)^{1 / 2}\left(\frac{\mathrm{GeV} / c^{2}}{m}\right)^{3 / 2} .
$$

Finally, the value of the mass-radius ratio $2 G M / R c^{2}=$ $2.95\left(M / M_{\odot}\right)(\mathrm{km} / R)$ of general relativistic BEC stars at the critical point is 0.319 in the fully-relativistic model, 0.529 in the semi-relativistic model, and 0.429 in the partially-relativistic model. It varies between $\sim 0.3$ and $\sim 0.5$ depending on the model. The mass-radius ratio is plotted as a function of $M / M_{*}$ in Fig. [6 for the different models. We note that the value of $2 G M / R c^{2}$ at the critical point provides the maximum value of the mass-radius ratio for the stable part of the series of equilibria.

The observations of neutron stars compiled by Mukherjee et al. [113] give a value of the mass-radius ratio $2 G M / R c^{2} \sim 0.5$ (see Table【). This is substantially larger

\footnotetext{
${ }^{2}$ We note that $R_{\min }$ and $\left(\epsilon_{0}\right)_{\max }$ depend only on $M_{\max }$ as a result of relativity. Furthermore, $M_{\max }$ depends only on $m$ and $a_{s}$ through the ratio $\kappa \propto a_{s}^{1 / 2} / m^{3 / 2}$. Therefore, there is only one free parameter $\kappa$ in the theory.
} 
than the value 0.319 predicted from the fully relativistic equation of state (A15). In other words, the predicted radius of the neutron stars is larger than observed. This led Mukherjee et al. 113 to conclude that the BEC model is ruled out. However, their conclusion may be too pessimistic because several effects can alter the equation of state of the BEC. For example, as they note, the interior of neutron stars could be a composition of BECs of kaons or pions. This may change the mass-radius relation of neutron stars. On the other hand, we note that the value 0.5 is relatively close to the values 0.429 and 0.529 obtained from the equations of state A25 and A29. This agreement is puzzling because these equations of state are less-well justified theoretically than the equation of state $\mathrm{A} 15$. This may be a further motivation to study these equations of state, independently of the BEC model. We finally note that the value 0.369 obtained in [117] is relatively close to the value 0.319 of the fully relativistic BEC model. Additional observations may be necessary to reach definite conclusions.

\section{On the maximum mass of neutron stars}

In their seminal paper, Oppenheimer and Volkoff [87] modeled neutron stars as a completely degenerate ideal gas of relativistic fermions. In that case, gravitational collapse is prevented by the Pauli exclusion principle. Since these objects are very compact, one must use general relativity. Therefore, the equilibrium configurations of neutron stars in this model are obtained by solving the TOV equations (27) and (28) with the equation of state $P(\epsilon)$ corresponding to a relativistic fermionic gas at $T=0$. This equation of state is given in parametric form by [100]:

$$
\begin{gathered}
P=A f(x), \quad \rho c^{2}=8 A x^{3}, \\
\epsilon=\rho c^{2}+E_{k i n}=8 A\left[x^{3}+\frac{1}{8} g(x)\right], \quad A=\frac{\pi m_{n}^{4} c^{5}}{3 h^{3}}, \\
f(x)=x\left(2 x^{2}-3\right)\left(x^{2}+1\right)^{1 / 2}+3 \sinh ^{-1} x, \\
g(x)=8 x^{3}\left[\left(x^{2}+1\right)^{1 / 2}-1\right]-f(x) .
\end{gathered}
$$

In the non-relativistic limit $(\epsilon \rightarrow 0)$, we get

$$
\begin{gathered}
\epsilon \sim \rho c^{2}, \quad P \sim \frac{1}{5}\left(\frac{3}{8 \pi}\right)^{2 / 3} \frac{h^{2}}{m_{n}^{8 / 3} c^{10 / 3}} \epsilon^{5 / 3}, \\
P \sim \frac{1}{5}\left(\frac{3}{8 \pi}\right)^{2 / 3} \frac{h^{2}}{m_{n}^{8 / 3}} \rho^{5 / 3}
\end{gathered}
$$

corresponding to a polytrope $n=3 / 2$. In the ultrarelativistic limit $(\epsilon \rightarrow+\infty)$, we get

$$
\begin{array}{r}
\epsilon \sim \frac{3}{4}\left(\frac{3}{8 \pi}\right)^{1 / 3} \frac{h c}{m_{n}^{4 / 3}} \rho^{4 / 3}, \quad P \sim \frac{1}{3} \epsilon, \\
P \sim \frac{1}{4}\left(\frac{3}{8 \pi}\right)^{1 / 3} \frac{h c}{m_{n}^{4 / 3}} \rho^{4 / 3},
\end{array}
$$

corresponding to a polytrope $n=3$. In this limit, the equation of state is linear: $P \sim \epsilon / 3$.

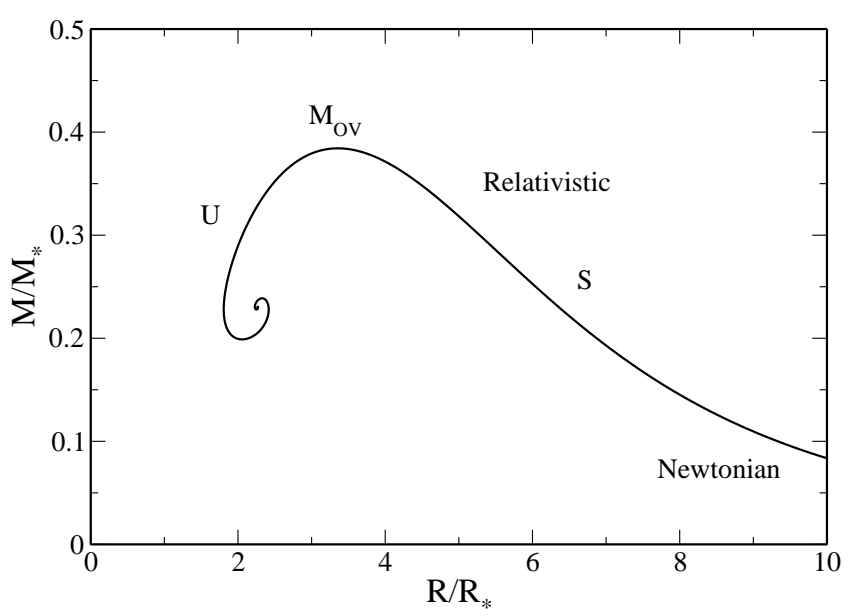

FIG. 7: Dimensionless mass-radius relation of neutron stars interpreted as fermion stars (Oppenheimer-Volkoff model) 87]. There exists a maximum mass $M_{O V}=0.384 M_{*}$, a minimum radius $R_{O V}=3.36 R_{*}$, and a maximum central energy density $\epsilon_{O V} / c^{2}=2.33 \times 10^{-2} \epsilon_{*}$ with $M_{*}=(\hbar c / G)^{3 / 2} / m_{n}^{2}$, $R_{*}=G M_{*} / c^{2}=\left(\hbar^{3} / G c\right)^{1 / 2} / m_{n}^{2}$ and $\epsilon_{*} / c^{2}=M_{*} / R_{*}^{3}=$ $m^{4} r^{3} / \hbar^{3}$

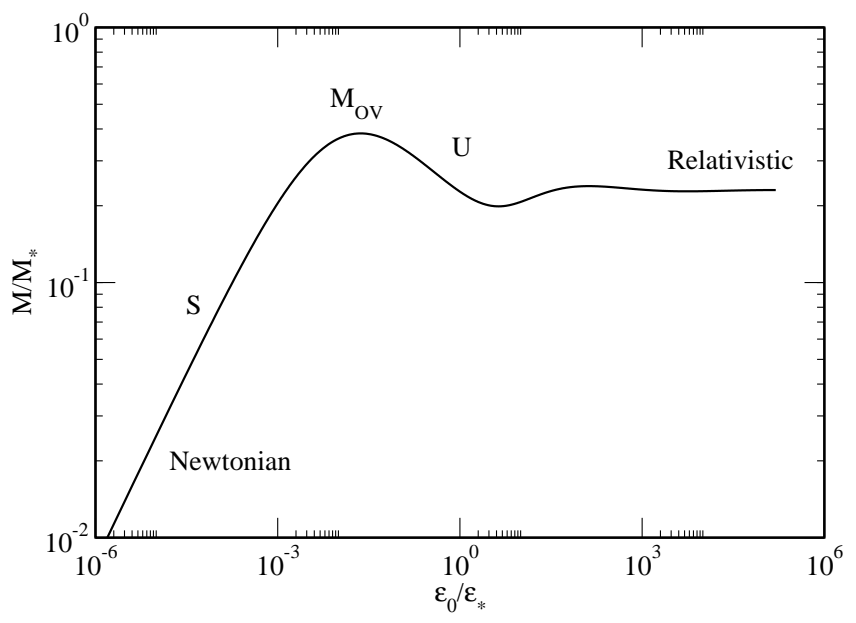

FIG. 8: Dimensionless mass-central energy density relation of neutron stars interpreted as fermion stars (OppenheimerVolkoff model) [87].

The mass-central density relation of fermion stars presents damped oscillations and the mass-radius relation has a snail-like (spiral) structure (see Figs. 7 and 
8) 87, 102 105. The maximum mass, minimum radius, and maximum energy density are ${ }^{3}$

$$
\begin{gathered}
M_{\mathrm{OV}}=0.384\left(\frac{\hbar c}{G}\right)^{3 / 2} \frac{1}{m_{n}^{2}}=0.709 M_{\odot} \\
R_{\mathrm{OV}}=8.735 \frac{G M_{O V}}{c^{2}}=9.15 \mathrm{~km} \\
\left(\epsilon_{c}\right)_{\mathrm{OV}} / c^{2}=3.44 \times 10^{-3} \frac{c^{6}}{G^{3} M_{O V}^{2}}=4.22 \times 10^{15} \mathrm{~g} / \mathrm{cm}^{3} .
\end{gathered}
$$

The mass-radius ratio at the critical point is

$$
\frac{2 G M_{\mathrm{OV}}}{R_{\mathrm{OV}} c^{2}}=0.229
$$

In this fermionic model, the maximum mass of a neutron star is determined by fundamental constants and by the mass $m_{n}$ of the neutrons. As a result, there is no indetermination and the maximum mass predicted by Oppenheimer and Volkoff [87] has a well-specified value $M_{\mathrm{OV}}=0.7 M_{\odot}$.

However, neutron stars with a mass in the range $2-2.4 M_{\odot}$, well above the Oppenheimer-Volkoff limit, have recently been observed [88 92]. These observations question the validity of the fermionic model. Therefore, alternative models of neutron stars should be constructed. In this respect, Chavanis and Harko [86] have proposed that, because of their superfluid cores, neutron stars could actually be BEC stars. Indeed, the neutrons (fermions) could form Cooper pairs and behave as bosons of mass $m=2 m_{n}$, where $m_{n}=0.940 \mathrm{GeV} / c^{2}$ is the mass of the neutrons. They can then make a BEC through the BCS/BEC crossover mechanism. Since the maximum mass of BEC stars $M_{\max }=0.307 \hbar c^{2} \sqrt{a_{s}} /(G m)^{3 / 2}$ [86] depends on the scattering length $a_{s}$ that is not wellknown, it can be larger than the Oppenheimer-Volkoff limit $M_{O V}=0.376 M_{P}^{3} / m_{n}^{2}=0.7 M_{\odot}$ [87] obtained by assuming that neutron stars can be modeled as an ideal gas of fermions. By taking a scattering length of the order of $10-20 \mathrm{fm}$ (giving $\kappa \sim 3.16-4.47$ ), we obtain a maximum mass of the order of $2 M_{\odot}$, a central density of the order $1-3 \times 10^{15} \mathrm{~g} / \mathrm{cm}^{3}$, and a radius in the range $10-20 \mathrm{~km}$ [86]. This could account for the recently observed neutron stars with masses in the range $2-2.4 M_{\odot}$ larger than the Oppenheimer-Volkoff limit. For $M<M_{\max }$, there exist stable equilibrium states of

\footnotetext{
${ }^{3}$ We note that Tooper [96], in his Sec. IX.b, considers a simplified model of neutron stars by using the non-relativistic equation of state for fermions $P=(1 / 5)(3 / 8 \pi)^{2 / 3} h^{2} / m_{n}^{8 / 3} \rho^{5 / 3}$ (corresponding to a polytrope of index $n=3 / 2)$ with the relativistic relation $\epsilon=\rho c^{2}+(3 / 2) P$ between the energy density and the rest-mass density. This is the fermionic counterpart of the partially-relativistic model of BEC stars (see Appendix A3).
}

BEC stars with $R>R_{\text {min }}$ for which gravitational collapse is prevented by the pressure arising from the scattering length of the bosons. For $M>M_{\max }$, nothing prevents the gravitational collapse of the star that becomes a black hole.

It is interesting to come back to the analogies and differences between fermion and boson stars (in the fullyrelativistic model of [86]). In the ultra-relativistic limit, they are both described by a polytropic equation of state $P \sim K^{\prime} \rho^{4 / 3}$ corresponding to an index $n=3$ but the polytropic constant is different. In the case of fermions $K^{\prime}=(1 / 4)(3 / 8 \pi)^{1 / 3} h c / m^{4 / 3}$ and in the case of bosons $K^{\prime}=(\pi / 8)^{1 / 3} \hbar^{2 / 3} a_{s}^{1 / 3} c^{4 / 3} / m$ (see Appendix A 2). In the two cases, the relation between the pressure and the energy density is $P \sim \epsilon / 3$. This is the relation that enters in the TOV equation. This linear equation of state is responsible for the damped oscillations of the mass-central density relation and for the snail-like structure (spiral) of the mass-radius relation. In the non-relativistic limit, fermion stars are described by a polytropic equation of state $P \sim K \rho^{5 / 3}$ of index $n=3 / 2$. The polytropic constant is $K=(1 / 5)(3 / 8 \pi)^{2 / 3} h^{2} / m^{8 / 3}$. This leads to the mass-radius relation $M R^{3}=1.49 \times 10^{-3} h^{6} /\left(G^{3} m^{8}\right)$. Therefore, there exist configurations of arbitrarily large radius and arbitrarily small mass (see Fig. 7). By contrast, in the non-relativistic limit, BEC stars are described by a polytropic equation of state $P=K \rho^{2}$ of in$\operatorname{dex} n=1$. The polytropic constant is $K=2 \pi a_{s} \hbar^{2} / m^{3}$. This fixes the radius of the configuration to the value $R=\pi\left(a_{s} \hbar^{2} / G m^{3}\right)^{1 / 2}$. Therefore, there is no configuration of radius larger than this value (see Fig. 5). This is a difference between fermion stars and BEC stars. On the other hand, in the case of fermion stars, the equation of state depends (apart from fundamental constants) only on the mass $m$ of the fermions. For neutron stars, this is the mass of the neutrons $m_{n}$ whose value is perfectly known. Therefore, the maximum mass of neutron stars modeled as fermion stars has an unambiguous value $0.7 M_{\odot}$. By contrast, in the case of BEC stars, the equation of state depends on $m$ and $a_{s}$ through the combination $\kappa^{2} \propto a_{s} / m^{3}$. As a result, the maximum mass of neutron stars modeled as BEC stars depends on this parameter $\kappa$ (compare Eqs. (50) and (62)). Since the value of this parameter is not well-known, it may be possible to overcome the Oppenheimer-Volkoff limit.

\section{E. On the maximum mass of baryon stars}

Zel'dovich [97] considered a gas of baryons interacting through a vector meson field and showed that the equation of state of this system is of the form of Eq. (32) with a polytropic constant

$$
K=\frac{g^{2} h^{2}}{2 \pi m_{m}^{2} m_{b}^{2} c^{2}},
$$

where $g$ is the baryon charge, $m_{m}$ is the meson mass, and $m_{b}$ is the baryon mass. Zel'dovich [97] introduced this 
equation of state as an example to show how the speed of sound could approach the speed of light at very high pressures and densities (see Appendix A 3.).

The equation of state (32) has been studied by Tooper [96] in relation to baryon stars (see his Sec. IX.c). Our treatment is a little more accurate and provides the following values for the maximum mass, minimum radius, and maximum density of baryon stars

$$
\begin{gathered}
M_{\max }=0.4104 \frac{g \hbar c}{m_{m} m_{b} G^{3 / 2}}=3.80 M_{\odot}, \\
R_{\min }=1.914 \frac{g \hbar}{m_{m} m_{b} c G^{1 / 2}}=26.2 \mathrm{~km}, \\
\left(\rho_{0}\right)_{\max }=0.318 \frac{m_{m}^{2} m_{b}^{2} c^{4}}{2 \pi g^{2} \hbar^{2}}=3.64 \times 10^{14} \mathrm{~g} / \mathrm{cm}^{3} .
\end{gathered}
$$

To make the numerical application, we have taken $g^{2} / h c \sim 1, m_{b} \sim m_{n}$, and $m_{m}=m_{b} / 2$ [96].

Zel'dovich model is also considered in Sec. 4.2 of [103] in the case where the equation of state (32) is approximated by its asymptotic form $P=\epsilon$ valid at high densities, and the system is enclosed within a spherical box of radius $R$ to make its mass finite. In this simplified setting, it is found that the critical mass-radius ratio $2 G M / R c^{2}$ is equal to 0.544 instead of 0.429 . The agreement is relatively satisfying in view of the crudeness of the box model. Some analogies between stiff stars and black holes are pointed out in [103].

\section{COSMOLOGY OF A BEC FLUID WITH A STIFF EQUATION OF STATE}

Harko [98] and Chavanis [64] considered the possibility that dark matter is made of BECs with a self-interaction and independently studied the cosmological implications of this model. ${ }^{4}$ If dark matter is made of BECs, it has a non-vanishing pressure even at $T=0$, unlike the CDM model. This affects the evolution of the scale factor of the universe. In most applications, the pressure arises from the self-interaction (the quantum pressure due to the Heisenberg uncertainty principle is negligible) so we can make the TF approximation. Harko 98] and Chavanis 64] consider a polytropic equation of state of the form of Eq. A29 and solve the corresponding Friedmann equations. However, this equation of state is not valid in the strongly relativistic regime so the extrapolation of their results to the very early universe is not correct. ${ }^{5}$ In this section, we solve the Friedmann equations

\footnotetext{
${ }^{4}$ Harko [98] considers repulsive self-interactions while Chavanis [64] considers repulsive and attractive self-interactions.

${ }^{5}$ Note that the equation of state A29 interesting in its own right in cosmology. Generalized polytropic equations of state of
}

with the equation of state A25. It leads to very different results in the early universe showing that the precise form of the equation of state of the BEC is crucial in cosmology. ${ }^{6}$ We stress that the equation of state A25 is itself not exact so the results of this section should be considered with caution. However, it is interesting to compare the effect of different equations of state on the evolution of the universe. Furthermore, the equation of state A25 interesting because it leads to a cosmological model involving a stiff matter phase. The same stiff matter phase occurs in the cosmological model of Zel'dovich [99] where the early universe is assumed to be made of a gas of cold baryons. The comparison between the different equations of state of BEC dark matter is made in Sec. IVD.

\section{A. The Friedmann equations}

We assume that the universe is homogeneous and isotropic, and contains a uniform perfect fluid of energy density $\epsilon(t)$ and isotropic pressure $P(t)$. The radius of curvature of the 3 -dimensional space, or scale factor, is noted $a(t)$ and the curvature of space is noted $k$. The universe is closed if $k>0$, flat if $k=0$, and open if $k<0$. We assume that the universe is flat $(k=0)$ in agreement with the observations of the cosmic microwave background (CMB) [122]. In that case, the Einstein equations can be written as [124]:

$$
\begin{gathered}
\frac{d \epsilon}{d t}+3 \frac{\dot{a}}{a}(\epsilon+P)=0 \\
\frac{\ddot{a}}{a}=-\frac{4 \pi G}{3 c^{2}}(\epsilon+3 P)+\frac{\Lambda}{3} \\
H^{2}=\left(\frac{\dot{a}}{a}\right)^{2}=\frac{8 \pi G}{3 c^{2}} \epsilon+\frac{\Lambda}{3},
\end{gathered}
$$

where we have introduced the Hubble parameter $H=$ $\dot{a} / a$ and accounted for a possible non-zero cosmological constant $\Lambda$. The cosmological constant is equivalent to a fluid with a constant energy density (dark energy):

$$
\epsilon_{\Lambda}=\rho_{\Lambda} c^{2}=\frac{\Lambda c^{2}}{8 \pi G}
$$

the form $P=\alpha \epsilon+k \epsilon^{1+1 / n}$ have been studied in full generality in Refs. [118 121]. For a negative polytropic pressure $(k<0)$, they lead to interesting cosmological models exhibiting a phase of early inflation and a phase of late accelerated expansion bridged by a phase of decelerating expansion. However, the justification of these equations of state may not be connected with BECs as initially thought.

6 It is less crucial in the context of BEC stars since the precise form of the equation of state only slightly changes the prefactor of the maximum mass (see Sec. IIIC). 
and an equation of state $P=-\epsilon$. Eqs. (70)-(72) are the well-known Friedmann equations describing a nonstatic universe. Among these three equations, only two are independent. The first equation can be viewed as an equation of continuity. For a given barotropic equation of state $P=P(\epsilon)$, it determines the relation between the energy density $\epsilon$ and the scale factor $a$. Then, the evolution of the scale factor $a(t)$ is given by Eq. (72).

\section{B. The equation of state of a partially-relativistic BEC fluid}

We assume that dark matter is made of a fluid at $T=0$ with an equation of state $P(\rho)$. In that case, the relation between the energy density $\epsilon$ and the mass density $\rho$ is given by the first law of relativistic thermodynamics (see Appendix A 1):

$$
d \epsilon=\frac{P+\epsilon}{\rho} d \rho .
$$

Combining this relation with the continuity equation (70), we get

$$
\frac{d \rho}{d t}+3 \frac{\dot{a}}{a} \rho=0
$$

We note that this equation is exact for a fluid at $T=0$ and that it does not depend on the explicit form of the equation of state $P(\rho)$. It can be integrated into

$$
\rho=\rho_{0}\left(\frac{a_{0}}{a}\right)^{3},
$$

where $\rho_{0}$ is the present value of the mass density and $a_{0}$ is the present value of the scale factor.

We now assume that dark matter is made of BECs at $T=0$ described by the equation of state

$$
P=K \rho^{2},
$$

where $K$ is given by Eq. (10). This polytropic equation of state of index $n=1$ corresponds to the partiallyrelativistic model of Appendix A3. The equation of state (77), with a polytropic constant $K$ given by Eq. (66), also appears in the cosmological model of Zel'dovich [99] where the early universe is assumed to be made of a cold gas of baryons. For the equation of state (77), Eq. (174) can be integrated easily and the relation between the energy density and the rest-mass density is given by

$$
\epsilon=\rho c^{2}+K \rho^{2} .
$$

Combining Eqs. (76) and (78), we get

$$
\epsilon=\rho_{0} c^{2}\left(\frac{a_{0}}{a}\right)^{3}+K \rho_{0}^{2}\left(\frac{a_{0}}{a}\right)^{6} .
$$

This relation can also be obtained by solving the continuity equation (70) with the equation of state A25 as detailed in Appendix D.
In the early universe $(a \rightarrow 0)$, we have

$$
\epsilon \sim K \rho_{0}^{2}\left(\frac{a_{0}}{a}\right)^{6}, \quad \epsilon \sim K \rho^{2}, \quad P \sim \epsilon
$$

These equations describe a stiff fluid $(P=\epsilon)$ for which the velocity of sound is equal to the velocity of light.

In the late universe $(a \rightarrow+\infty)$, we have

$$
\epsilon \sim \rho_{0} c^{2}\left(\frac{a_{0}}{a}\right)^{3}, \quad \epsilon \sim \rho c^{2}, \quad P \sim \frac{K}{c^{4}} \epsilon^{2} .
$$

These equations describe a classical BEC fluid with a polytropic equation of state of index $n=1(P=$ $\left.K \epsilon^{2} / c^{4}\right)$. Actually, for very large values of the scale factor, we recover the results of the CDM model $(P=0)$ since $\epsilon \propto a^{-3}$.

\section{The solution of the Friedmann equation for a partially-relativistic BEC fluid}

Substituting Eq. (79) in the Friedmann equation (72), we get

$$
\left(\frac{\dot{a}}{a}\right)^{2}=\frac{8 \pi G}{3 c^{2}}\left[\rho_{0} c^{2}\left(\frac{a_{0}}{a}\right)^{3}+K \rho_{0}^{2}\left(\frac{a_{0}}{a}\right)^{6}+\rho_{\Lambda} c^{2}\right] .
$$

This first order differential equation determines the temporal evolution of the scale factor $a(t)$. Its formal solution is

$$
\int_{0}^{a(t) / a_{0}} \frac{d x}{x \sqrt{\frac{\rho_{0} c^{2}}{x^{3}}+\frac{K \rho_{0}^{2}}{x^{6}}+\rho_{\Lambda} c^{2}}}=\left(\frac{8 \pi G}{3 c^{2}}\right)^{1 / 2} t
$$

This equation has the same form as Eq. (E5) obtained under the assumption that the universe is made of three non-interacting fluids corresponding to stiff matter, dust matter, and dark energy. Therefore, we can immediately transpose the results of Appendix E to the present context. The universe starts from a primordial singularity and it successively undergoes a stiff matter phase $\left(\epsilon \propto a^{-6}\right)$, a dust matter phase $\left(\epsilon \propto a^{-3}\right)$, and a dark energy phase $\left(\epsilon \sim \rho_{\Lambda} c^{2}\right)$.

The evolution of the scale factor is explicitly given by

$$
\begin{array}{r}
\frac{a}{a_{0}}=\left[\left(\frac{\rho_{0}}{\rho_{\Lambda}}+2 \sqrt{\frac{K \rho_{0}^{2}}{\rho_{\Lambda} c^{2}}}\right) \sinh ^{2}\left(\sqrt{6 \pi G \rho_{\Lambda}} t\right)\right. \\
\left.+\sqrt{\frac{K \rho_{0}^{2}}{\rho_{\Lambda} c^{2}}}\left(1-e^{-2 \sqrt{6 \pi G \rho_{\Lambda}} t}\right)\right]^{1 / 3} .
\end{array}
$$

The evolution of the energy density is given by

$$
\begin{aligned}
\epsilon & =\left[\left(\frac{1}{2} \sqrt{\frac{\rho_{0}^{2} c^{2}}{\rho_{\Lambda}}}+\sqrt{K \rho_{0}^{2}}\right) \sinh \left(2 \sqrt{6 \pi G \rho_{\Lambda}} t\right)\right. \\
& \left.+\sqrt{K \rho_{0}^{2}} e^{-2 \sqrt{6 \pi G \rho_{\Lambda}} t}\right]^{2} /\left(\frac{a}{a_{0}}\right)^{6} .
\end{aligned}
$$


This is a simple generalization of the $\Lambda \mathrm{CDM}$ model for a BEC universe assumed to be described by the equation of state (77). For $t \rightarrow+\infty$, we recover the de Sitter solution

$$
\frac{a}{a_{0}} \sim\left(\frac{\rho_{0}}{\rho_{\Lambda}}+2 \sqrt{\frac{K \rho_{0}^{2}}{\rho_{\Lambda} c^{2}}}\right)^{1 / 3} \frac{1}{2^{2 / 3}} e^{\sqrt{\frac{8 \pi G \rho_{\Lambda}}{3}} t}
$$

with a prefactor affected by the BEC.

In the absence of a cosmological constant $\left(\rho_{\Lambda}=0\right)$, the solution of Eq. (82) is

$$
\begin{aligned}
\frac{a}{a_{0}} & =\left(6 \pi G \rho_{0} t^{2}+2 \sqrt{\frac{6 \pi G K \rho_{0}^{2}}{c^{2}}} t\right)^{1 / 3}, \\
\epsilon & =\frac{c^{2}}{6 \pi G t^{2}}\left(\frac{1+\sqrt{\frac{K}{6 \pi G}} \frac{1}{c t}}{1+2 \sqrt{\frac{K}{6 \pi G}} \frac{1}{c t}}\right)^{2} .
\end{aligned}
$$

This is a simple generalization of the Einstein-de Sitter (EdS) model for a BEC universe assumed to be described by the equation of state (77).

Returning to the case $\Lambda \geq 0$ and considering the formal limit $K \rightarrow+\infty$, equivalent to the case of Appendix E where we can neglect dust matter in front of stiff matter, we get

$$
\begin{gathered}
\frac{a}{a_{0}}=\left(\frac{K \rho_{0}^{2}}{\rho_{\Lambda} c^{2}}\right)^{1 / 6} \sinh ^{1 / 3}\left(2 \sqrt{6 \pi G \rho_{\Lambda}} t\right), \\
\epsilon=\frac{\rho_{\Lambda} c^{2}}{\sinh ^{2}\left(2 \sqrt{6 \pi G \rho_{\Lambda}} t\right)} .
\end{gathered}
$$

In the absence of cosmological constant $(\Lambda=0)$, the foregoing equations reduce to

$$
\frac{a}{a_{0}}=\left(2 \sqrt{\frac{6 \pi G K \rho_{0}^{2}}{c^{2}}} t\right)^{1 / 3}, \quad \epsilon=\frac{c^{2}}{24 \pi G t^{2}} .
$$

We also recover well-known models as particular cases of the foregoing equations. In the absence of BECs $(K=$ 0 ), we recover the $\Lambda \mathrm{CDM}$ model

$$
\begin{gathered}
\frac{a}{a_{0}}=\left(\frac{\rho_{0}}{\rho_{\Lambda}}\right)^{1 / 3} \sinh ^{2 / 3}\left(\sqrt{6 \pi G \rho_{\Lambda}} t\right), \\
\epsilon=\frac{\rho_{\Lambda} c^{2}}{\sinh ^{2}\left(\sqrt{6 \pi G \rho_{\Lambda}} t\right)} .
\end{gathered}
$$

In the absence of BECs and cosmological constant $(K=$ $\Lambda=0$ ), we recover the EdS universe

$$
\frac{a}{a_{0}}=\left(6 \pi G \rho_{0} t^{2}\right)^{1 / 3}, \quad \epsilon=\frac{c^{2}}{6 \pi G t^{2}} .
$$

The previous results have been presented in the context of a dark matter fluid made of BECs with an equation of state given by Eq. (77). As discussed in the next section, this model may give wrong results for BECs in the early universe because it is based on a classical equation of state. However, the equation of state (77) also appears in the cosmological model of Zel'dovich [99] where the early universe is assumed to be made of a cold gas of baryons. This model presents a stiff matter phase that follows the cosmological singularity (Big Bang). In that context, the equation of state (77) is rigorously justified. Therefore, the analytical solutions that we have presented in this section are exact in the context of Zel'dovich's model 99]. Actually, Zel'dovich [99] briefly mentions that the complete equation of state in his model is of the form

$$
P=K \rho^{2}+K^{\prime} \rho^{4 / 3},
$$

where $K$ is given by Eq. (66) and the second term accounts for quantum (Fermi) corrections. For the equation of state (95), we find from Eq. (A14) that the relation between the energy density and the rest-mass density is

$$
\epsilon=\rho c^{2}+K \rho^{2}+3 K^{\prime} \rho^{4 / 3} .
$$

Substituting Eq. (76) in Eq. (96), we obtain

$$
\epsilon=\rho_{0} c^{2}\left(\frac{a_{0}}{a}\right)^{3}+K \rho_{0}^{2}\left(\frac{a_{0}}{a}\right)^{6}+3 K^{\prime} \rho_{0}^{4 / 3}\left(\frac{a_{0}}{a}\right)^{4} .
$$

When combined with the Friedmann equation (172), we obtain a model of universe exhibiting a stiff matter phase $\left(\epsilon \propto a^{-6}\right)$, a radiation phase $\left(\epsilon \propto a^{-4}\right)$, a dust matter phase $\left(\epsilon \propto a^{-3}\right)$, and a dark energy phase $\left(\epsilon \sim \rho_{\Lambda} c^{2}\right)$ as discussed in Appendix E

Remark: In this paper, we have considered the case of a repulsive self-interaction $(K \geq 0)$. The case of an attractive self-interaction $(K<0)$ is treated in [123]. In that case, the primordial universe is non-singular. We have also assumed that the cosmological constant is positive in agreement with the observations. The case of a negative cosmological constant (anti-de Sitter), leading to an oscillatory universe, is considered in [123].

\section{Comparison between the different models of BEC cosmology}

We now compare the different models of BEC cosmology depending on the considered equation of state.

Harko 98] and Chavanis [64] assumed that the BEC dark matter is described by an equation of state of the form A29) and solved the corresponding Friedmann equations. This corresponds to the semi-relativistic model of Appendix A4. For a repulsive self-interaction $\left(a_{s}>0\right)$ they found that the universe starts with a new form of singularity in which the energy density is infinite while the scale factor is finite. At sufficiently late times, the universe returns the usual $\Lambda \mathrm{CDM}$ model in which the universe experiences an EdS phase $\left(a \propto t^{2 / 3}, \epsilon \propto t^{-2}\right)$ 
followed by a de Sitter phase $\left(a \propto e^{\sqrt{\Lambda / 3} t}, \epsilon=\rho_{\Lambda} c^{2}\right)$. However, this model differs from the $\Lambda \mathrm{CDM}$ model in the intermediate phase because of the contribution of the BEC. In particular, it is found that the scale factor increases more rapidly when dark matter is made of BEC instead of pressureless matter [64, 98].

In this paper, we have assumed that the BEC dark matter is described by the equation of state (A22). This corresponds to the partially-relativistic model of Appendix A3 Solving the corresponding Friedmann equation, we have found that the early universe behaves as a stiff fluid $(P \sim \epsilon)$. The scale factor increases as $a \propto t^{1 / 3}$ while the energy density decreases as $\epsilon \propto t^{-2}$. The universe starts from a singularity at $t=0$ in which the energy density is infinite while the scale factor vanishes. At later times, the universe behaves as a nonrelativistic $\mathrm{BEC}$ with an equation of state $P \sim K \epsilon^{2} / c^{4}$.

We stress, however, that the previous models may be incorrect in the very early universe because they use an approximate relativistic equation of state. A better model of BEC dark matter should be based on the equation of state (A15). This corresponds to the fullyrelativistic model of Appendix A2 In that case, the early universe has an equation of state $P \sim \epsilon / 3$ similar to the equation of state of radiation. At later times, the universe behaves as a nonrelativistic BEC with an equation of state $P \sim K \epsilon^{2} / c^{4}$.

Remark: Although the evolution of the early universe is very sensitive to the equation of state of the BEC, it should be recalled that BECs do form only when the temperature has sufficiently decreased. Therefore, we should be careful when extrapolating the solutions to the past. If we view BEC dark matter as a small correction to pressureless matter $(\Lambda \mathrm{CDM}$ model), all the equations of state of Appendix $\mathrm{A}$ reduce to $P \sim K \epsilon^{2} / c^{4}$ and give equivalent results for sufficiently late times. In this sense, the cosmological models of Harko [98] and Chavanis [64] are justified for sufficiently late times after the primordial singularity and after the appearance of BECs.

\section{CONCLUSION}

In this paper, we have compared different models of relativistic self-gravitating BECs.

Concerning general relativistic BEC stars, we have shown that the partially-relativistic model of Appendix A 3 (leading to a stiff equation of state) gives a maximum mass that is smaller than the semi-relativistic model of Appendix A4 but larger than the fully-relativistic model of Appendix A2 However, the difference is relatively small (the main indetermination of the maximum mass being the value of the scattering length of the particles) so that the three models provide a fair description of general relativistic BEC stars. Of course, the fully relativistic treatment is the most relevant one on a physical point of view. However, the observed mass-radius ratio of neutron stars seems to be closer to the value obtained from the partially-relativistic model or from the value obtained from the semi-relativistic model than to the value obtained from the fully-relativistic model. Therefore, the equations of state A25) and (A29) may be useful to model neutron stars, independently of the BEC model. In this respect, we can note that they are special cases of the two polytropic models developed by Tooper [95, 96] for an index $n=1$. However, more observations may be necessary to determine a precise value of the mass-radius ratio of neutron stars and ascertain these conclusions.

Concerning the evolution of a universe made of BEC dark matter, the precise form of the equation of state is crucial in the very early universe. If the dark matter is described by the equation of state (A29), corresponding to the semi-relativistic model, the universe starts from a primordial singularity in which the scale factor is finite and the density is infinite. On the other hand, if the dark matter is described by the equation of state (A25) corresponding to the partially relativistic model, the early universe undergoes a stiff matter era in which the scale factor increases as $a \propto t^{1 / 3}$ and the energy density decreases as $\epsilon \propto a^{-6}$. Finally, if the dark matter is described by the equation of state A15 corresponding to the fully relativistic model, the early universe undergoes a radiation era in which the scale factor increases as $a \propto t^{1 / 2}$ and the energy density decreases as $\epsilon \propto a^{-4}$. In principle, only the fully-relativistic model is relevant in the very early universe. However, at later times, all the models give equivalent results.

The stiff equation of state A25 also describes a baryon star or the primordial evolution of a universe filled with a cold gas of baryons as proposed by Zel'dovich [97, 99]. Therefore, the results that we obtained with this equation of state can have application in the context of cold baryons (Zel'dovich model), independently of the BEC model.

\section{Appendix A: The equation of state of a relativistic BEC}

\section{General results}

The local form of the first law of thermodynamics can be expressed as

$$
d\left(\frac{\epsilon}{\rho}\right)=-P d\left(\frac{1}{\rho}\right)+T d\left(\frac{s}{\rho}\right),
$$

where $\rho=n m$ is the mass density, $n$ is the number density, and $s$ is the entropy density in the rest frame. For a system at $T=0$, the first law of thermodynamics reduces to

$$
d \epsilon=\frac{P+\epsilon}{\rho} d \rho .
$$

For a given equation of state, Eq. A2 can be integrated to obtain the relation between the energy density $\epsilon$ and the rest-mass density $\rho$. 
If the equation of state is prescribed under the form $P=P(\epsilon)$, Eq. A2 can be immediately integrated into

$$
\ln \rho=\int \frac{d \epsilon}{P(\epsilon)+\epsilon} .
$$

If, as an example, we consider the "gamma law" equation of state [125, 126]:

$$
P=(\gamma-1) \epsilon
$$

we get

$$
P=K \rho^{\gamma}, \quad \epsilon=\frac{K}{\gamma-1} \rho^{\gamma},
$$

where $K$ is a constant of integration.

We now assume that the equation of state is prescribed under the form $P=P(\rho)$. In that case, Eq. A2 reduces to the first order linear differential equation

$$
\frac{d \epsilon}{d \rho}-\frac{1}{\rho} \epsilon=\frac{P(\rho)}{\rho} .
$$

Using the method of the variation of the constant, we obtain

$$
\epsilon=A \rho c^{2}+\rho \int^{\rho} \frac{P\left(\rho^{\prime}\right)}{\rho^{\prime 2}} d \rho^{\prime},
$$

where $A$ is a constant of integration.

As an example, we consider the polytropic equation of state [100]:

$$
P=K \rho^{\gamma}, \quad \gamma=1+\frac{1}{n}
$$

For $\gamma=1$, we get

$$
\epsilon=A \rho c^{2}+K \rho \ln \rho .
$$

For $\gamma \neq 1$, we obtain

$$
\epsilon=A \rho c^{2}+\frac{K}{\gamma-1} \rho^{\gamma}=A \rho c^{2}+n P .
$$

Taking $A=0$, we recover Eqs. A4 - A5 . We now assume $0<n<+\infty$ (i.e. $\gamma>1$ ). In that case, we determine the constant $A$ by requiring that $\epsilon \sim \rho c^{2}$ when $\rho \rightarrow 0$. This gives $A=1$. As a result, Eq. A10 takes the form

$$
\epsilon=\rho c^{2}+\frac{K}{\gamma-1} \rho^{\gamma}=\rho c^{2}+n P .
$$

For $\rho \rightarrow 0$ (non-relativistic limit), we get

$$
\epsilon \sim \rho c^{2}, \quad P \sim K\left(\epsilon / c^{2}\right)^{\gamma}
$$

For $\rho \rightarrow+\infty$ (ultra-relativistic limit), we get

$$
\epsilon \sim n K \rho^{\gamma}, \quad P \sim \epsilon / n \sim(\gamma-1) \epsilon .
$$

For a general equation of state $P(\rho)$ such that $P \sim \rho^{\gamma}$ with $\gamma>1$ when $\rho \rightarrow 0$, we determine the constant $A$ in Eq. (A7) by requiring that $\epsilon \sim \rho c^{2}$ when $\rho \rightarrow 0$. This gives

$$
\epsilon=\rho c^{2}+\rho \int_{0}^{\rho} \frac{P\left(\rho^{\prime}\right)}{\rho^{\prime 2}} d \rho^{\prime}=\rho c^{2}+u(\rho) .
$$

We note that $\rho c^{2}$ is the rest mass energy density and $u(\rho)$ may be interpreted as an internal energy [11].

\section{Fully-relativistic model}

We consider the equation of state

$$
P=\frac{c^{4}}{36 K}\left(\sqrt{1+\frac{12 K}{c^{4}}} \epsilon-1\right)^{2},
$$

where $K$ is given by Eq. (10). This equation of state can be derived from the relativistic Klein-Gordon equation of a self-interacting scalar field in the strong coupling limit 69]. It also applies to a relativistic self-interacting $\mathrm{BEC}$ at $T=0$ in the $\mathrm{TF}$ approximation [86]. It provides a fully-relativistic BEC model. For $\epsilon \rightarrow 0$ (nonrelativistic limit), we recover the polytropic equation of state $P=K\left(\epsilon / c^{2}\right)^{2}$ of a classical BEC. For $\epsilon \rightarrow+\infty$ (ultra-relativistic limit), we obtain a linear equation of state $P=\epsilon / 3$ similar to the one describing the core of neutron stars modeled by the ideal Fermi gas (see Sec. IIID 87, 102 105.

For the equation of state (A15), Eq. (A3) becomes

$$
\frac{1}{3} \ln \rho=\int^{12 K \epsilon / c^{4}} \frac{d x}{(\sqrt{1+x}-1)^{2}+3 x} .
$$

Using the identity

$$
\begin{array}{r}
\int \frac{d x}{(\sqrt{1+x}-1)^{2}+3 x}=\frac{1}{3} \ln (\sqrt{1+x}-1) \\
+\frac{1}{6} \ln (1+2 \sqrt{1+x}),
\end{array}
$$

and requiring that $\epsilon \sim \rho c^{2}$ for $\rho \rightarrow 0$, we obtain the following relation between the rest-mass density and the energy density

$\rho=\frac{c^{2}}{6 \sqrt{3} K}\left(\sqrt{1+\frac{12 K}{c^{4}}} \epsilon-1\right)\left[1+2 \sqrt{1+\frac{12 K}{c^{4}}} \epsilon\right]^{1 / 2}$.

For $\rho \rightarrow 0$ (non-relativistic limit), we get

$$
\epsilon \sim \rho c^{2}, \quad P \sim \frac{K}{c^{4}} \epsilon^{2}, \quad P \sim K \rho^{2},
$$

corresponding to a polytrope $n=1$. This returns the equation of state (8) of a classical BEC. For $\rho \rightarrow+\infty$ 
(ultra-relativistic limit), we get

$$
\epsilon \sim \frac{3 c^{4 / 3} K^{1 / 3}}{2^{4 / 3}} \rho^{4 / 3}, \quad P \sim \frac{1}{3} \epsilon, \quad P \sim \frac{c^{4 / 3} K^{1 / 3}}{2^{4 / 3}} \rho^{4 / 3},
$$

corresponding to a polytrope $n=3$. This is similar to the equation of state of an ultra-relativistic Fermi gas at $T=0$ (core of neutron star) but the polytropic constant is different (see Sec. IIID).

For the equation of state (A15), the velocity of sound is given by

$$
\frac{c_{s}^{2}}{c^{2}}=P^{\prime}(\epsilon)=\frac{1}{3}\left(1-\frac{1}{\sqrt{1+12 K \epsilon / c^{4}}}\right) .
$$

We always have $c_{s}<c$. For $\epsilon \rightarrow+\infty, c_{s} \rightarrow c / \sqrt{3}$.

\section{Partially-relativistic model}

We consider the equation of state

$$
P=K \rho^{2},
$$

where $K$ is given by Eq. (10). This equation of state can be derived from the classical GP equation. It describes a non-relativistic self-interacting BEC at $T=0$ in the TF approximation. We assume that this relation remains valid in the relativistic regime. This is not exact but it provides a partially-relativistic BEC model.

Since the equation of state A22 corresponds to a polytrope $n=1$, Eq. (A11) reduces to

$$
\epsilon=\rho c^{2}+P=\rho c^{2}+K \rho^{2} .
$$

This equation can be reversed to give

$$
\rho=\frac{c^{2}}{2 K}\left(\sqrt{1+\frac{4 K \epsilon}{c^{4}}}-1\right) .
$$

Combining Eqs. A22 and A24, we obtain the relation between the pressure and the energy density

$$
P=\frac{c^{4}}{4 K}\left(\sqrt{1+\frac{4 K \epsilon}{c^{4}}}-1\right)^{2} .
$$

This equation of state has a form similar to Eq. A15 but the coefficients are different (see Appendix C). We note that Eq. A22 with Eq. A23 is a particular case of the class of equations of state studied by Tooper 96 in general relativity. For $\epsilon \rightarrow 0$ (non-relativistic limit), we recover the polytropic equation of state $P=K\left(\epsilon / c^{2}\right)^{2}$ of a classical BEC. For $\epsilon \rightarrow+\infty$ (ultra-relativistic limit), we obtain a linear equation of state $P=\epsilon$. This is a stiff equation of state in which the velocity of sound is equal to the velocity of light $\left(c_{s}=c\right)$. This type of equations of state has been introduced by Zel'dovich [97] in the context of baryon stars in which the baryons interact through a vector meson field (see Sec. IIIE).

For $\rho \rightarrow 0$ (non-relativistic limit), we get

$$
\epsilon \sim \rho c^{2}, \quad P \sim \frac{K}{c^{4}} \epsilon^{2}, \quad P=K \rho^{2} .
$$

For $\rho \rightarrow+\infty$ (ultra-relativistic limit), we get

$$
\epsilon \sim K \rho^{2}, \quad P \sim \epsilon, \quad P=K \rho^{2} .
$$

For the equation of state (A15), the velocity of sound is given by

$$
\frac{c_{s}^{2}}{c^{2}}=P^{\prime}(\epsilon)=1-\frac{1}{\sqrt{1+4 K \epsilon / c^{4}}} .
$$

We always have $c_{s} \leq c$. For $\epsilon \rightarrow+\infty, c_{s} \rightarrow c$.

\section{Semi-relativistic model}

We consider the equation of state

$$
P=\frac{K}{c^{4}} \epsilon^{2},
$$

where $K$ is given by Eq. (10). This equation of state was studied as a preliminary model by Chavanis and Harko [86] before treating the fully-relativistic model corresponding to the equation of state A15. The equation of state (A29) is obtained from Eq. (8), derived from the classical GP equation, by replacing the rest mass density by the energy density (i.e. by making the approximation $\epsilon \sim \rho c^{2}$ ), and by assuming that the resulting equation remains valid in the relativistic regime. This is not exact but it provides a semi-relativistic BEC model. We note that Eq. A29) is a particular case of the class of polytropic equations of state studied by Tooper [95] in general relativity.

For the equation of state (A29), Eq. (A3) becomes

$$
\ln \rho=\int \frac{d \epsilon}{\left(\frac{K \epsilon}{c^{4}}+1\right) \epsilon} .
$$

Performing the integral and requiring that $\epsilon \sim \rho c^{2}$ for $\rho \rightarrow 0$, we obtain the following relation between the mass density and the energy density

$$
\rho=\frac{\epsilon / c^{2}}{\frac{K \epsilon}{c^{4}}+1} .
$$

This equation can be reversed to give

$$
\epsilon=\frac{\rho c^{2}}{1-\frac{K \rho}{c^{2}}} .
$$

Combining Eqs. (A29) and (A32) we obtain

$$
P=\frac{K \rho^{2}}{\left(1-K \rho / c^{2}\right)^{2}} .
$$


We note that the pressure diverges when $\rho=c^{2} / K$. Therefore, there is a maximum density

$$
\rho_{\max }=\frac{c^{2}}{K}=\frac{m^{3} c^{2}}{2 \pi a_{s} \hbar^{2}} .
$$

For $\rho \rightarrow 0$ (non-relativistic regime), we get

$$
\epsilon \sim \rho c^{2}, \quad P \sim \frac{K}{c^{4}} \epsilon^{2}, \quad P \sim K \rho^{2} .
$$

For $\rho \rightarrow c^{2} / K$, we get

$$
\epsilon \sim \frac{c^{4} / K}{1-\frac{K \rho}{c^{2}}}, \quad P \sim \frac{K}{c^{4}} \epsilon^{2}, \quad P \sim \frac{c^{4} / K}{\left(1-K \rho / c^{2}\right)^{2}} .
$$

For the equation of state (A29), the velocity of sound is given by

$$
c_{s}^{2}=\frac{2 K}{c^{2}} \epsilon .
$$

The velocity of sound can be mathematically larger than the velocity of light but such configurations are dynamically unstable [86].

Remark: For a general polytropic equation of state of the form $P=K\left(\epsilon / c^{2}\right)^{\gamma}$ we get

$$
\rho=\frac{\epsilon / c^{2}}{\left[1+\frac{K}{c^{2 \gamma}} \epsilon^{\gamma-1}\right]^{\frac{1}{\gamma-1}}}, \quad \epsilon=\frac{\rho c^{2}}{\left(1-\frac{K}{c^{2}} \rho^{\gamma-1}\right)^{\frac{1}{\gamma-1}}},
$$

and

$$
P=\frac{K \rho^{\gamma}}{\left(1-\frac{K}{c^{2}} \rho^{\gamma-1}\right)^{\frac{\gamma}{\gamma-1}}}
$$

\section{Appendix B: The Newtonian value of the maximum mass of a relativistic BEC star}

It is interesting to compare the value of the maximum mass of a relativistic BEC star obtained by using general relativity (see [86]) with the one obtained by using Newtonian gravity. We consider the fully relativistic equation of state of Appendix A 2. In general relativity, we have to substitute in the TOV equations (27) and (28) the equation of state A15 relating the pressure $P$ to the energy density $\epsilon$. This leads to the maximum mass of Eq. (50) with $A_{2}=0.307$ 86]. In Newtonian gravity, we have to substitute in the equation of hydrostatic equilibrium (13) the equation of state defined by Eqs. A15 and A18 relating the pressure $P$ to the mass density $\rho$. In order to determine the maximum mass of a relativistic BEC star in the Newtonian framework, it is sufficient to consider the limiting form of this equation of state in the ultrarelativistic limit $\rho \rightarrow+\infty .7^{7}$ Eq. A20 can be rewritten

\footnotetext{
7 We use a treatment similar to the one performed by Chandrasekar [127] in the case of relativistic white dwarf stars treated with Newtonian gravity.
}

as

$$
P=\frac{\pi^{1 / 3} c^{4 / 3} \hbar^{2 / 3} a_{s}^{1 / 3}}{2 m} \rho^{4 / 3}
$$

This is the equation of state of a polytrope of index $n=3$ [100]. Combining the condition of hydrostatic equilibrium (13) with the Poisson equation (6), we obtain

$$
\nabla \cdot\left(\frac{\nabla P}{\rho}\right)=-4 \pi G \rho .
$$

Substituting the equation of state (B1) in Eq. (B2), we get

$$
\frac{c^{4 / 3} \hbar^{2 / 3} a_{s}^{1 / 3}}{2 \pi^{2 / 3} G m} \Delta \rho^{1 / 3}=-\rho
$$

Defining

$$
\rho=\rho_{0} \theta^{3}, \quad \xi=\left(\frac{2 \pi^{2 / 3} G m \rho_{0}^{2 / 3}}{c^{4 / 3} \hbar^{2 / 3} a_{s}^{1 / 3}}\right)^{1 / 2} r
$$

where $\rho_{0}$ is the central density, we obtain the LaneEmden equation

$$
\frac{1}{\xi^{2}} \frac{d}{d \xi}\left(\xi^{2} \frac{d \theta}{d \xi}\right)=-\theta^{3},
$$

$$
\theta(0)=1, \quad \theta^{\prime}(0)=0
$$

for a polytrope of index $n=3$ [100]. This equation has to be solved numerically. The function $\theta(\xi)$ vanishes at $\xi_{1}=6.89685$. At that point $\omega_{3} \equiv-\xi_{1}^{2} \theta^{\prime}\left(\xi_{1}\right)=2.01824$. The mass of the star $M=\int_{0}^{R} \rho 4 \pi r^{2} d r$ is given by

$$
M=\sqrt{2} \omega_{3} \frac{\hbar c^{2} \sqrt{a_{s}}}{(G m)^{3 / 2}},
$$

with $\sqrt{2} \omega_{3}=2.854$. We note that the radius $R$ of a star described by the equation of state B1 can take arbitrary values. The relation between the radius and the central density of the star is

$$
R=\frac{\xi_{1}}{\sqrt{2} \pi^{1 / 3}} \frac{c^{2 / 3} \hbar^{1 / 3} a_{s}^{1 / 6}}{m^{1 / 2} G^{1 / 2} \rho_{0}^{1 / 3}},
$$

with $\xi_{1} / \sqrt{2} \pi^{1 / 3}=3.33$. We have the relation

$$
\rho_{0} R^{3}=\frac{\xi_{1}^{3}}{4 \pi \omega_{3}} M
$$

with $\xi_{1}^{3} / 4 \pi \omega_{3}=12.9$. The mean density of the star $\bar{\rho}=$ $3 M / 4 \pi R^{3}$ is related to the central density by the relation $\bar{\rho}=3 \omega_{3} \rho_{0} / \xi_{1}^{3}=1.85 \times 10^{-2} \rho_{0}$.

It is interesting to contrast the calculations of this Appendix to those of Sec. IIB. For a polytrope of index 
$n=1$, the radius of the star is fixed but its mass is unspecified. Inversely, for a polytrope of index $n=3$, the mass of the star is fixed but its radius is unspecified. For other values of the polytropic index, the radius of the star is a function of its mass. The general mass-radius relation of a polytropic star with an equation of state $P=K \rho^{1+1 / n}$ is [100]:

$$
M^{(n-1) / n} R^{(3-n) / n}=\frac{K(n+1)}{G(4 \pi)^{1 / n}} \omega_{n}^{(n-1) / n},
$$

where $\omega_{n}=-\xi_{1}^{(n+1) /(n-1)} \theta^{\prime}\left(\xi_{1}\right)$. For $n=1$ and $n=3$, we recover the results of Sec. IIB and of the present Appendix.

When the full equation of state $P(\rho)$ defined by Eqs. A15 and A18 is considered, we find that the limiting configuration corresponding to the ultra-relativistic limit determined by the equation of state (B1) has a radius $R=0$ and an infinite density $\rho_{0} \rightarrow+\infty$ [55]. This configuration corresponds to a Dirac peak of mass $M$. Therefore, the mass defined by Eq. (B7) corresponds to the maximum mass of a relativistic BEC star in Newtonian gravity. It has the correct scaling of Eq. (22) but the prefactor 2.854 is very different from the exact prefactor 0.307 obtained in general relativity. This shows that general relativity is crucial to determine the maximum mass of relativistic BEC stars. The same conclusion is reached for neutron stars considered as fermion stars. The general relativistic approach of Oppenheimer and Volkoff 87 leads to a maximum mass equal to $M_{\max }=0.376(\hbar c / G)^{3 / 2} m_{n}^{-2}=0.7 M_{\odot}$ while the Newtonian treatment of Chandrasekhar leads to a maximum mass equal to $M_{\max }^{\prime}=3.10(\hbar c / G)^{3 / 2} m_{n}^{-2}=5.76 M_{\odot}$ 127, 128.

\section{Appendix C: Generalized equation of state and} alternative form of the differential equation (36)-(37)

Let us consider the equation of state

$$
P=\frac{q^{2} c^{4}}{4 K}\left(\sqrt{1+\frac{4 K}{q c^{4}}} \epsilon-1\right)^{2}
$$

When $\epsilon \rightarrow 0$ (non-relativistic regime) we recover the quadratic equation of state $P \sim K \epsilon^{2} / c^{4}$ of a classical BEC. When $\epsilon \rightarrow+\infty$ (ultra-relativistic regime) we obtain a linear equation of state $P \sim q \epsilon$. The equation of state (C1) generalizes the equations of state A15 and (A25) corresponding to $q=1 / 3$ and $q=1$ respectively.

The relation between the rest-mass density and the energy density is given by Eq. A3 which can be rewritten as

$$
q \ln \rho=\int^{4 K \epsilon / q c^{4}} \frac{d x}{(\sqrt{1+x}-1)^{2}+\frac{x}{q}} .
$$

The integral can be calculated analytically. Setting $y=$ $\sqrt{x+1}$, we obtain

$$
\int \frac{d x}{(\sqrt{1+x}-1)^{2}+\frac{x}{q}}=\frac{A}{B}
$$

with

$$
\begin{array}{r}
A=q(y-1)[1+y+q(y-1)] \\
\times\{(1+q) \ln (y-1)-(q-1) \ln [1+y+q(y-1)]\}
\end{array}
$$

and

$$
B=(1+q)[x+q(2+x-2 y)] .
$$

For $q=1 / 3$, we recover Eq. (A17), and for $q=1$ the expression in the right hand side of Eq. (C2) reduces to $\ln (y-1)$ returning the equations of Appendix A3 obtained the other way round.

In Sec. IIIB, we have written the TOV equations associated to the equation of state (32), equivalent to Eq. (33), by introducing the variable $\theta$ defined with the restmass density $\rho$. Alternatively, we can introduce a variable $\Theta$ defined with the energy density $\epsilon$ as in [86] (be careful to the change of notations). If we set

$$
\begin{gathered}
\epsilon=\epsilon_{0} \Theta, \quad r=\frac{\xi}{A}, \quad \sigma=\frac{K \epsilon_{0}}{c^{4}}, \\
M(r)=\frac{4 \pi \epsilon_{0}}{A^{3} c^{2}} v(\xi), \quad A=\left(\frac{2 \pi G}{K}\right)^{1 / 2},
\end{gathered}
$$

and substitute the equation of state (C1) in the TOV equations (27) and (28), using

$$
P^{\prime}(\epsilon)=q\left[1-\frac{1}{\sqrt{1+4 K \epsilon / q c^{4}}}\right],
$$

we obtain

$$
\begin{gathered}
\frac{d \Theta}{d \xi}=-\frac{\frac{2}{q}\left[\frac{q^{2}}{4}(\sqrt{1+4 \sigma \Theta / q}-1)^{2}+\sigma \Theta\right]\left[v+\frac{q^{2} \xi^{3}}{4 \sigma}(\sqrt{1+4 \sigma \Theta / q}-1)^{2}\right]}{\xi^{2}(1-4 \sigma v / \xi)(1-1 / \sqrt{1+4 \sigma \Theta / q})}, \\
\frac{d v}{d \xi}=\Theta \xi^{2}
\end{gathered}
$$


For $q=1 / 3$ these equations reduce to Eqs. (87) and (88) of [86].

\section{Appendix D: Alternative derivation of Eq. (79)}

In this Appendix, we check that Eq. (79) can be obtained directly from the equation of continuity (70) with the equation of state A25).

Substituting Eq. (A25) in Eq. (170), and simplifying some terms, we get

$$
\frac{2 K}{c^{4}} \frac{d \epsilon}{d a}+\frac{3}{a}\left(\frac{4 K \epsilon}{c^{4}}+1-\sqrt{1+\frac{4 K \epsilon}{c^{4}}}\right)=0 .
$$

With the change of variables $x=\left(1+4 K \epsilon / c^{4}\right)^{1 / 2}$, we obtain

$$
\frac{d x}{d a}+\frac{3}{a}(x-1)=0
$$

This equation can be integrated into

$$
x=1+\frac{A}{a^{3}},
$$

where $A$ is a constant. Returning to original variables, we obtain

$$
\epsilon=\frac{c^{4}}{4 K}\left(\frac{2 A}{a^{3}}+\frac{A^{2}}{a^{6}}\right),
$$

which can be written as Eq. (79).

\section{Appendix E: A universe with a stiff matter era}

In this Appendix, we assume that the universe is made of one or several fluids each of them described by a linear equation of state $P=\alpha \epsilon$. The equation of continuity (70) implies that the energy density is related to the scale factor by $\epsilon=\epsilon_{0}\left(a_{0} / a\right)^{3(1+\alpha)}$, where the subscript 0 denotes present-day values of the quantities. A linear equation of state can describe dust matter $\left(\alpha=0, \epsilon_{m} \propto a^{-3}\right)$, radiation $\left(\alpha=1 / 3, \epsilon_{\text {rad }} \propto a^{-4}\right)$, stiff matter $(\alpha=1$, $\left.\epsilon_{s} \propto a^{-6}\right)$, vacuum energy $\left(\alpha=-1, \epsilon=\epsilon_{P}\right)$, and dark energy $\left(\alpha=-1, \epsilon=\epsilon_{\Lambda}\right)$.

We consider a universe made of stiff matter, radiation, dust matter and dark energy treated as non-interacting species. Summing the contribution of each species, the total energy density can by written as

$$
\epsilon=\frac{\epsilon_{s, 0}}{\left(a / a_{0}\right)^{6}}+\frac{\epsilon_{\text {rad }, 0}}{\left(a / a_{0}\right)^{4}}+\frac{\epsilon_{m, 0}}{\left(a / a_{0}\right)^{3}}+\epsilon_{\Lambda} .
$$

In this model, the stiff matter dominates in the early universe. This is followed by the radiation era, by the dust matter era and, finally, by the dark energy era. Writing $\epsilon_{\alpha, 0}=\Omega_{\alpha, 0} \epsilon_{0}$ for each species, we get

$$
\frac{\epsilon}{\epsilon_{0}}=\frac{\Omega_{s, 0}}{\left(a / a_{0}\right)^{6}}+\frac{\Omega_{r a d, 0}}{\left(a / a_{0}\right)^{4}}+\frac{\Omega_{m, 0}}{\left(a / a_{0}\right)^{3}}+\Omega_{\Lambda, 0} .
$$

The energy density starts from $\epsilon=+\infty$ at $a=0$, decreases, and tends to $\epsilon_{\Lambda}$ for $a \rightarrow+\infty$. The relation between the energy density and the scale factor is shown in Fig. 9. The proportions of stiff matter, dust matter and dark energy as a function of the scale factor are shown in Fig. 10. Using Eq. (E2), the Friedmann equation (72) takes the form

$$
\frac{H}{H_{0}}=\sqrt{\frac{\Omega_{s, 0}}{\left(a / a_{0}\right)^{6}}+\frac{\Omega_{r a d, 0}}{\left(a / a_{0}\right)^{4}}+\frac{\Omega_{m, 0}}{\left(a / a_{0}\right)^{3}}+\Omega_{\Lambda, 0}}
$$

with $\Omega_{s, 0}+\Omega_{\text {rad }, 0}+\Omega_{m, 0}+\Omega_{\Lambda, 0}=1$ and $H_{0}=$ $\left(8 \pi G \epsilon_{0} / 3 c^{2}\right)^{1 / 2}$. Note that we have taken $\Lambda=0$ in Eq. (72) and accounted for the effect of the cosmological constant in the dark energy density $\epsilon_{\Lambda}$. We also note the relation

$$
\frac{\epsilon}{\epsilon_{0}}=\left(\frac{H}{H_{0}}\right)^{2}
$$

that will be used later. The evolution of the scale factor is given by

$$
\int_{0}^{a / a_{0}} \frac{d x}{x \sqrt{\frac{\Omega_{s, 0}}{x^{6}}+\frac{\Omega_{r a d, 0}}{x^{4}}+\frac{\Omega_{m, 0}}{x^{3}}+\Omega_{\Lambda, 0}}}=H_{0} t .
$$

We first ignore radiation $\left(\Omega_{r a d, 0}=0\right)$ and consider a universe made of stiff matter, dust matter, and dark energy. In that case, the Friedmann equation (E5) reduces to

$$
\int_{0}^{a / a_{0}} \frac{d x}{x \sqrt{\frac{\Omega_{s, 0}}{x^{6}}+\frac{\Omega_{m, 0}}{x^{3}}+\Omega_{\Lambda, 0}}}=H_{0} t
$$

Using the identity

$$
\begin{gathered}
\int \frac{d x}{x \sqrt{\frac{a}{x^{3}}+\frac{b}{x^{6}}+c}} \\
=\frac{1}{3 \sqrt{c}} \ln \left[a+2 c x^{3}+2 \sqrt{c} \sqrt{b+a x^{3}+c x^{6}}\right],
\end{gathered}
$$

Eq. (E6) can be solved analytically to give

$$
\begin{aligned}
\frac{a}{a_{0}}=\left[\left(\frac{\Omega_{m, 0}}{\Omega_{\Lambda, 0}}\right.\right. & \left.+2 \sqrt{\frac{\Omega_{s, 0}}{\Omega_{\Lambda, 0}}}\right) \sinh ^{2}\left(\frac{3}{2} \sqrt{\Omega_{\Lambda, 0}} H_{0} t\right) \\
& \left.+\sqrt{\frac{\Omega_{s, 0}}{\Omega_{\Lambda, 0}}}\left(1-e^{-3 \sqrt{\Omega_{\Lambda, 0}}} H_{0} t\right)\right]^{1 / 3} .
\end{aligned}
$$

From Eq. (E8), we can compute $H=\dot{a} / a$ leading to

$$
\begin{aligned}
\left(\frac{a}{a_{0}}\right)^{3} \frac{H}{H_{0}} & =\left(\frac{\Omega_{m, 0}}{2 \sqrt{\Omega_{\Lambda, 0}}}+\sqrt{\Omega_{s, 0}}\right) \sinh \left(3 \sqrt{\Omega_{\Lambda, 0}} H_{0} t\right) \\
& +\sqrt{\Omega_{s, 0}} e^{-3 \sqrt{\Omega_{\Lambda, 0}} H_{0} t} .
\end{aligned}
$$

The energy density is given by Eq. (E4) where $H / H_{0}$ can be obtained from (E9) with Eq. (E8). 
At $t=0$ the universe starts from a singular state at which the scale factor $a=0$ while the energy density $\epsilon=$ $+\infty$. The scale factor increases with time. For $t \rightarrow+\infty$, we obtain

$$
\frac{a}{a_{0}} \sim\left(\frac{\Omega_{m, 0}}{\Omega_{\Lambda, 0}}+2 \sqrt{\frac{\Omega_{s, 0}}{\Omega_{\Lambda, 0}}}\right)^{1 / 3} \frac{1}{2^{2 / 3}} e^{\sqrt{\Omega_{\Lambda, 0}}} H_{0} t .
$$

The energy density decreases with time and tends to $\epsilon_{\Lambda}$ for $t \rightarrow+\infty$. The expansion is decelerating during the stiff matter era and the dust matter era while it is accelerating during the dark energy era. The temporal evolution of the scale factor and of the energy density are shown in Fig. 11 and 12

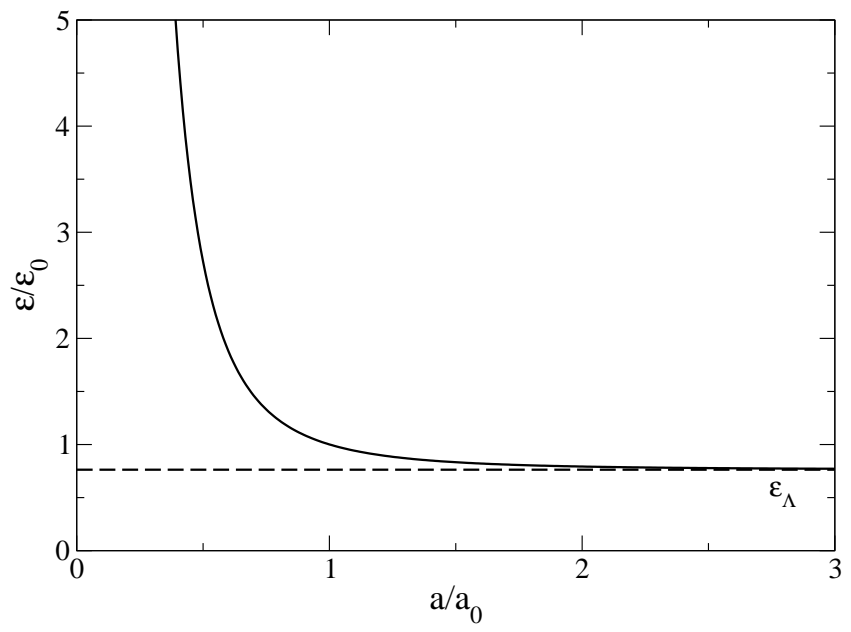

FIG. 9: Energy density as a function of the scale factor. We have taken $\Omega_{m, 0}=0.237, \Omega_{\Lambda, 0}=0.763$, and $\Omega_{s, 0}=10^{-3}$ (we have chosen a relatively large value of the density of stiff matter $\Omega_{s, 0}=10^{-3}$ for a better illustration of the results).

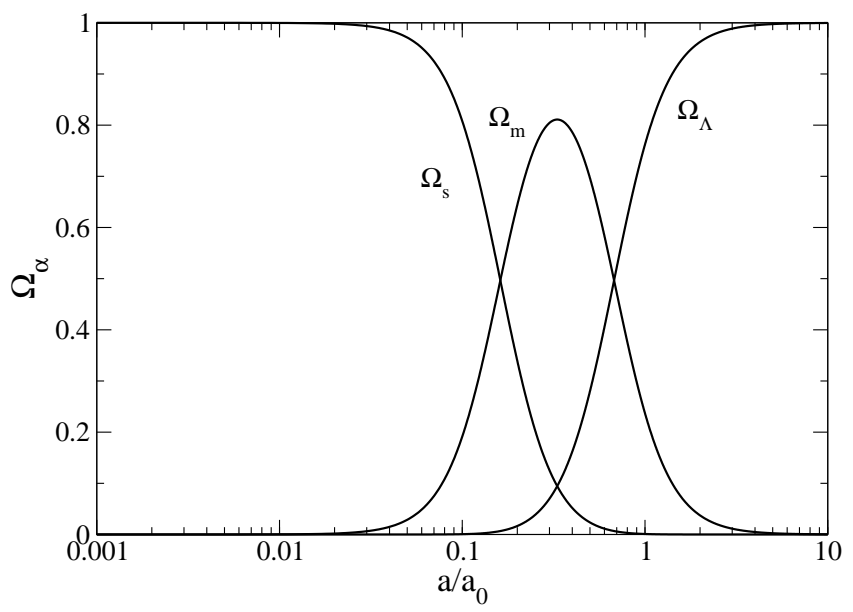

FIG. 10: Evolution of the proportion $\Omega_{\alpha}=\epsilon_{\alpha} / \epsilon$ of the different components of the universe with the scale factor.

We consider a universe made of stiff matter and dust matter. In the absence of dark energy $\left(\Omega_{\Lambda, 0}=0\right)$, using

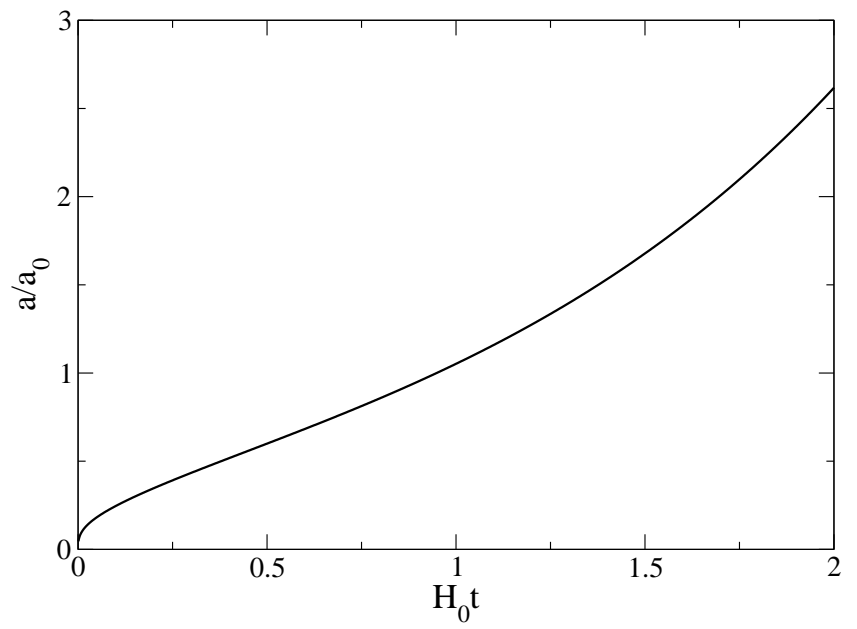

FIG. 11: Evolution of the scale factor as a function of time.

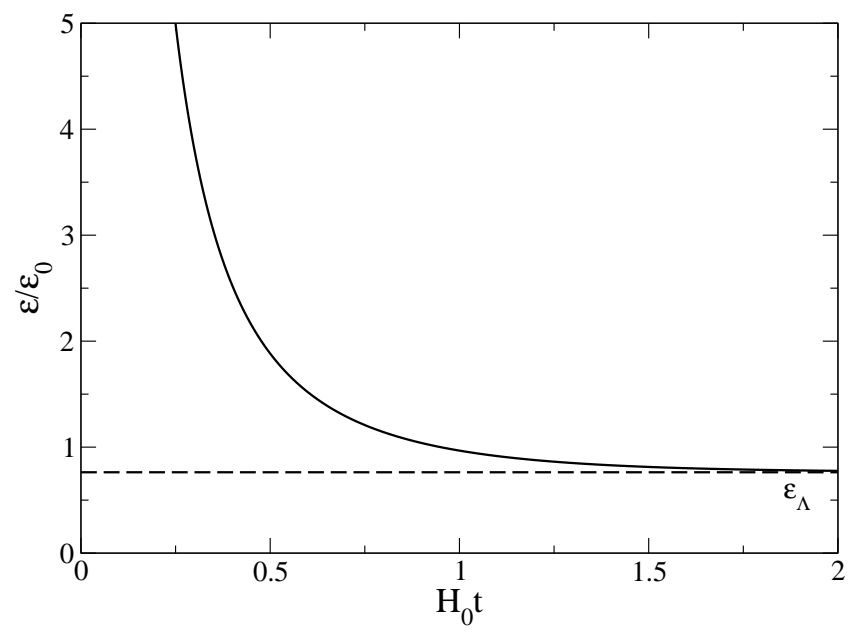

FIG. 12: Evolution of the energy density as a function of time.

the identity

$$
\int \frac{d x}{x \sqrt{\frac{a}{x^{3}}+\frac{b}{x^{6}}}}=\frac{2}{3 a} \sqrt{b+a x^{3}},
$$

we obtain

$$
\begin{gathered}
\frac{a}{a_{0}}=\left(\frac{9}{4} \Omega_{m, 0} H_{0}^{2} t^{2}+3 \sqrt{\Omega_{s, 0}} H_{0} t\right)^{1 / 3}, \\
\frac{\epsilon}{\epsilon_{0}}=\frac{4}{9 H_{0}^{2} t^{2}}\left(\frac{1+\frac{2 \sqrt{\Omega_{s, 0}}}{3 \Omega_{m, 0} H_{0} t}}{1+\frac{4 \sqrt{\Omega_{s, 0}}}{3 \Omega_{m, 0} H_{0} t}}\right)^{2} .
\end{gathered}
$$

We consider a universe made of stiff matter and dark energy. In the absence of matter $\left(\Omega_{m, 0}=0\right)$, using the identity

$$
\int \frac{d x}{x \sqrt{\frac{b}{x^{6}}+c}}=\frac{1}{3 \sqrt{c}} \ln \left[2 c x^{3}+2 \sqrt{c} \sqrt{b+c x^{6}}\right],
$$


or setting $X=b / c x^{6}$ and using the identity

$$
\int \frac{d X}{X \sqrt{X+1}}=\ln \left(\frac{\sqrt{1+X}-1}{\sqrt{1+X}+1}\right),
$$

we get

$$
\begin{gathered}
\frac{a}{a_{0}}=\left(\frac{\Omega_{s, 0}}{\Omega_{\Lambda, 0}}\right)^{1 / 6} \sinh ^{1 / 3}\left(3 \sqrt{\Omega_{\Lambda, 0}} H_{0} t\right), \\
\frac{\epsilon}{\epsilon_{0}}=\frac{\Omega_{\Lambda, 0}}{\tanh ^{2}\left(3 \sqrt{\Omega_{\Lambda, 0}} H_{0} t\right)} .
\end{gathered}
$$

We consider a universe made of stiff matter. In the absence of dust matter and dark energy $\left(\Omega_{m, 0}=\Omega_{\Lambda, 0}=\right.$ $0)$, we find that

$$
\frac{a}{a_{0}}=\left(3 \sqrt{\Omega_{s, 0}} H_{0} t\right)^{1 / 3}, \quad \frac{\epsilon}{\epsilon_{0}}=\frac{1}{9 H_{0}^{2} t^{2}} .
$$

We consider a universe made of dust matter and dark energy. In the absence of stiff matter $\left(\Omega_{s, 0}=0\right)$, using the identity

$$
\int \frac{d x}{x \sqrt{\frac{a}{x^{3}}+c}}=\frac{1}{3 \sqrt{c}} \ln \left[a+2 c x^{3}+2 \sqrt{c} \sqrt{a x^{3}+c x^{6}}\right],
$$

or setting $X=a / c x^{3}$ and using Eq. (E15), we obtain

$$
\begin{gathered}
\frac{a}{a_{0}}=\left(\frac{\Omega_{m, 0}}{\Omega_{\Lambda, 0}}\right)^{1 / 3} \sinh ^{2 / 3}\left(\frac{3}{2} \sqrt{\Omega_{\Lambda, 0}} H_{0} t\right), \\
\frac{\epsilon}{\epsilon_{0}}=\frac{\Omega_{\Lambda, 0}}{\tanh ^{2}\left(\frac{3}{2} \sqrt{\Omega_{\Lambda, 0}} H_{0} t\right)} .
\end{gathered}
$$

This solution coincides with the $\Lambda$ CDM model.

We consider a universe made of dark energy. In the absence of stiff matter and dust matter $\left(\Omega_{s, 0}=\Omega_{m, 0}=\right.$ $0)$, we obtain

$$
a(t)=a(0) e^{\sqrt{\frac{\Lambda}{3}} t}, \quad \epsilon=\epsilon_{\Lambda}
$$

This is de Sitter's solution.

We consider a universe made of dust matter. In the absence of stiff matter and dark energy $\left(\Omega_{s, 0}=\Omega_{\Lambda, 0}=\right.$ $0)$, we obtain

$$
\frac{a}{a_{0}}=\left(\frac{9}{4} \Omega_{m, 0} H_{0}^{2} t^{2}\right)^{1 / 3}, \quad \frac{\epsilon}{\epsilon_{0}}=\frac{4}{9 H_{0}^{2} t^{2}} .
$$

This is the Einstein-de Sitter (EdS) solution.

We now come back to the general equation (E3) including the contribution of radiation.

The transition between the stiff matter era and the radiation era is obtained by taking $\Omega_{m, 0}=\Omega_{\Lambda, 0}=0$ in
Eq. (E3). In that case, the integral in Eq. (E5) can be performed analytically leading to

$$
\begin{array}{r}
2 \sqrt{\Omega_{\text {rad }, 0}} \frac{a}{a_{0}} \sqrt{\Omega_{s, 0}+\Omega_{\text {rad }, 0}\left(\frac{a}{a_{0}}\right)^{2}}-2 \Omega_{s, 0} \\
\times \ln \left[\Omega_{\text {rad }, 0} \frac{a}{a_{0}}+\sqrt{\Omega_{\text {rad }, 0}} \sqrt{\Omega_{s, 0}+\Omega_{\text {rad }, 0}\left(\frac{a}{a_{0}}\right)^{2}}\right] \\
+\Omega_{s, 0} \ln \left(\Omega_{s, 0} \Omega_{r a d, 0}\right)=4\left(\Omega_{\text {rad }, 0}\right)^{3 / 2} H_{0} t .
\end{array}
$$

The transition between the radiation era and the matter era is obtained by taking $\Omega_{s, 0}=\Omega_{\Lambda, 0}=0$ in Eq. (E3). In that case, the integral in Eq. (E5) can be performed analytically leading to ${ }^{8}$

$$
\begin{array}{r}
H_{0} t=-\frac{2}{3} \frac{1}{\left(\Omega_{m, 0}\right)^{1 / 2}}\left(\frac{2 \Omega_{\text {rad }, 0}}{\Omega_{m, 0}}-\frac{a}{a_{0}}\right) \sqrt{\frac{\Omega_{\text {rad }, 0}}{\Omega_{m, 0}}+\frac{a}{a_{0}}} \\
+\frac{4}{3} \frac{\left(\Omega_{r a d, 0}\right)^{3 / 2}}{\left(\Omega_{m, 0}\right)^{2}} .
\end{array}
$$

Eq. E25 can also be written as

$$
\left(\frac{a}{a_{0}}\right)^{3}-3 \frac{\Omega_{r a d, 0}}{\Omega_{m, 0}}\left(\frac{a}{a_{0}}\right)^{2}=\frac{9}{4} \Omega_{m, 0} H_{0}^{2} t^{2}-6 \frac{\Omega_{r a d, 0}^{3 / 2}}{\Omega_{m, 0}} H_{0} t .
$$

This is a cubic equation for $a / a_{0}$.

For mathematical completeness, we also give the equations corresponding to a universe containing only radiation and dark energy $\left(\Omega_{s, 0}=\Omega_{m, 0}=0\right)$. They write

$$
\begin{gathered}
\frac{a}{a_{0}}=\left(\frac{\Omega_{r a d, 0}}{\Omega_{\Lambda, 0}}\right)^{1 / 4} \sinh ^{1 / 2}\left(2 \sqrt{\Omega_{\Lambda, 0}} H_{0} t\right), \\
\frac{\epsilon}{\epsilon_{0}}=\frac{\Omega_{\Lambda, 0}}{\tanh ^{2}\left(2 \sqrt{\Omega_{\Lambda, 0}} H_{0} t\right)} .
\end{gathered}
$$

For a universe containing only radiation $\left(\Omega_{s, 0}=\Omega_{m, 0}=\right.$ $\left.\Omega_{\Lambda, 0}=0\right)$ we get

$$
\frac{a}{a_{0}}=\Omega_{r a d, 0}^{1 / 4} \sqrt{2 H_{0} t}, \quad \frac{\epsilon}{\epsilon_{0}}=\frac{1}{\left(2 H_{0} t\right)^{2}} .
$$

Finally, we can propose a simple generalization of Eq. (E3) that includes a phase of early inflation. Using the arguments developed in [118, 119] we get

$$
\frac{H}{H_{0}}=\sqrt{\frac{\Omega_{s, 0}}{\left(a / a_{0}\right)^{6}+\left(a_{1} / a_{0}\right)^{6}}+\frac{\Omega_{\text {rad,0}}}{\left(a / a_{0}\right)^{4}}+\frac{\Omega_{m, 0}}{\left(a / a_{0}\right)^{3}}+\Omega_{\Lambda, 0}},
$$

\footnotetext{
${ }^{8}$ We have determined the constant of integration in Eq. E25 such that $a=0$ at $t=0$. This implicitly assumes that there is no stiff matter in the early universe. Otherwise, we need to determine the constant of integration by matching the solution E25 with the solution E18 of the stiff matter era.
} 
where the constant $a_{1}$ is determined by the relation $\epsilon_{P} a_{1}^{6}=\epsilon_{s, 0} a_{0}^{6}$ where $\epsilon_{P}=\rho_{P} c^{2}$ is the Planck energy density. The transition between the inflation era and the stiff matter era is obtained by taking $\Omega_{\text {rad }, 0}=\Omega_{m, 0}=$ $\Omega_{\Lambda, 0}=0$ in Eq. E30). In that case, Eq. E30 can be integrated analytically to give [118]:

$$
\sqrt{R^{6}+1}-\ln \left(\frac{1+\sqrt{R^{6}+1}}{R^{3}}\right)=3 K t+C
$$

where we have defined $R=a / a_{1}$ and $K=$ $H_{0}\left(\Omega_{s, 0} a_{0}^{6} / a_{1}^{6}\right)^{1 / 2}=\left(8 \pi G \rho_{P} / 3\right)^{1 / 2}$. $C$ is a constant of integration that can be determined by requiring that $a=l_{P}$ at $t=0$ where $l_{P}$ is the Planck length.
[1] C. J. Pethick, H. Smith, Bose-Einstein condensation in dilute gases (Cambridge, Cambridge University Press, 2008)

[2] J.F. Navarro, C.S. Frenk, S.D.M. White, Mon. Not. R. astr. Soc. 462, 563 (1996)

[3] A. Burkert, Astrophys. J. 447, L25 (1995)

[4] P. Salucci, A. Burkert, Astrophys. J. 537, L9 (2000)

[5] G. Kauffmann, S.D.M. White, B. Guiderdoni, Mon. Not. R. astr. Soc. 264, 201 (1993)

[6] E.P. Gross, Ann. of Phys. 4, 57 (1958); Nuovo Cimento 20, 454 (1961); J. Math. Phys. 4, 195 (1963)

[7] L.P. Pitaevskii, Sov. Phys. JETP 9, 830 (1959); ibid 13, 451 (1961)

[8] E. Madelung, Zeit. F. Phys. 40, 322 (1927)

[9] P.J.E. Peebles, B. Ratra, Rev. Mod. Phys. 75, 559 (2003)

[10] A. Suárez, V.H. Robles, T. Matos, Astrophys. Space Sci. Proc. 38, 107 (2014)

[11] T. Rindler-Daller, P.R. Shapiro, Astrophys. Space Sci. Proc. 38, 163 (2014)

[12] P.H. Chavanis, Self-gravitating Bose-Einstein condensates, in Quantum Aspects of Black Holes, edited by X. Calmet (Springer, 2015)

[13] M.R. Baldeschi, G.B. Gelmini, R. Ruffini, Phys. Lett. B 122, 221 (1983)

[14] M. Membrado, A.F. Pacheco, J. Sanudo, Phys. Rev. A 39, 4207 (1989)

[15] S.J. Sin, Phys. Rev. D 50, 3650 (1994)

[16] F.E. Schunck, astro-ph/9802258

[17] T. Matos, F.S. Guzmán, F. Astron. Nachr. 320, 97 (1999)

[18] F.S. Guzmán, T. Matos, Class. Quantum Grav. 17, L9 (2000)

[19] W. Hu, R. Barkana, A. Gruzinov, Phys. Rev. Lett. 85, $1158(2000)$

[20] T. Matos, L.A. Ureña-López, Phys. Rev. D 63, 063506 (2001)

[21] A. Arbey, J. Lesgourgues, P. Salati, Phys. Rev. D 64, $123528(2001)$

[22] M.P. Silverman, R.L. Mallett, Class. Quantum Grav. 18, L103 (2001)

[23] M. Alcubierre, F.S. Guzmán, T. Matos, D. Núñez, L.A. Ureña-López, P. Wiederhold, Class. Quantum. Grav. 19, 5017 (2002)

[24] M.P. Silverman, R.L. Mallett, Gen. Rel. Grav. 34, 633 (2002)

[25] A. Bernal, T. Matos, D. Núñez, Rev. Mex. Astron. Astrofis. 44, 149 (2008)

[26] P. Sikivie, Q. Yang, Phys. Rev. Lett. 103, 111301 (2009)

[27] T. Matos, A. Vazquez-Gonzalez, J. Magana, Mon. Not.
R. Astron. Soc. 393, 1359 (2009)

[28] J.W. Lee, Phys. Lett. B 681, 118 (2009)

[29] T.P. Woo, T. Chiueh, Astrophys. J. 697, 850 (2009)

[30] J.W. Lee, S. Lim, J. Cosmol. Astropart. Phys. 01, 007 (2010)

[31] P.H. Chavanis, Phys. Rev. D 84, 043531 (2011)

[32] P.H. Chavanis, L. Delfini, Phys. Rev. D 84, 043532 (2011)

[33] G. Manfredi, P.A. Hervieux, F. Haas, Class. Quantum Grav. 30, 075006 (2013)

[34] H.Y. Schive, T. Chiueh, T. Broadhurst, Nature Physics 10, 496 (2014)

[35] H.Y. Schive et al. arXiv:1407.7762

[36] R. Ruffini, S. Bonazzola, Phys. Rev. 187, 1767 (1969)

[37] P.H. Chavanis, M. Lemou, F. Méhats, arXiv:1409.7840

[38] J.W. Lee, I. Koh, Phys. Rev. D 53, 2236 (1996)

[39] P.J.E. Peebles, Astrophys. J. 534, L127 (2000)

[40] J. Goodman, New Astronomy 5, 103 (2000)

[41] J. Lesgourgues, A. Arbey, P. Salati, New Astron. Rev. 46, 791 (2002)

[42] A. Arbey, J. Lesgourgues, P. Salati, Phys. Rev. D 68, 023511 (2003)

[43] C.G. Böhmer, T. Harko, J. Cosmol. Astropart. Phys. 06, 025 (2007)

[44] F. Briscese, Phys. Lett. B 696, 315 (2011)

[45] T. Harko, J. Cosmol. Astropart. Phys. 05, 022 (2011)

[46] M.O.C. Pires, J.C.C. de Souza, J. Cosmol. Astropart. Phys. 11 (2012) 024

[47] V.H. Robles, T. Matos, Monthly Not. Roy. Astron. Soc. 422, 282 (2012)

[48] T. Rindler-Daller, P. R. Shapiro, Monthly Not. Roy. Astron. Soc. 422, 135 (2012)

[49] V. Lora, J. Magaña, A. Bernal, F.J. Sánchez-Salcedo, E.K. Grebel, J. Cosmol. Astropart. Phys. 02, 011 (2012)

[50] A.X. González-Morales, A. Diez-Tejedor, L.A. UreñaLópez, O. Valenzuela, Phys. Rev. D 87, 021301(R) (2013)

[51] F.S. Guzmán, F.D. Lora-Clavijo, J.J. González-Avilés, F.J. Rivera-Paleo, J. Cosmol. Astropart. Phys. 09 (2013) 034

[52] F. Dalfovo, S. Giorgini, L.P. Pitaevskii, S. Stringari, Rev. Mod. Phys. 71, 463 (1999)

[53] S.W. Randall, M. Markevitch, D. Clowe, A.H. Gonzalez, M. Bradac, Astrophys. J. 679, 1173 (2008)

[54] T. Fukuyama, M. Morikawa, T. Tatekawa, J. Cosmol. Astropart. Phys. 06 (2008) 033

[55] P.H. Chavanis, preprint

[56] G. Ingrosso, R. Ruffini, Nuovo Cimento 101, 369 (1988)

[57] G. Ingrosso, M. Merafina, R. Ruffini, Nuovo Cimento 105, 977 (1990) 
[58] N. Bilic, H. Nikolic, Nucl. Phys. B 590, 575 (2000)

[59] T. Matos, A. Suárez, Europhys. Lett. 96, 56005 (2011)

[60] T. Harko, E. Madarassy, J. Cosmol. Astropart. Phys. 01, 020 (2012)

[61] Z. Slepian, J. Goodman, Mon. Not. R. Astron. Soc. 427, $839(2012)$

[62] T. Harko, G. Mocanu, Phys. Rev. D 85, 084012 (2012)

[63] V.H. Robles, T. Matos, Astrophys. J. 763, 19 (2013)

[64] P.H. Chavanis, Astron. Astrophys. 537, A127 (2012)

[65] D.J. Kaup, Phys. Rev. 172, 1331 (1968)

[66] W. Thirring, Phys. Lett. B 127, 27 (1983)

[67] J.D. Breit, S. Gupta, A. Zaks, Phys. Lett. B 140, 329 (1984)

[68] E. Takasugi, M. Yoshimura, Z. Phys. C 26, 241 (1984)

[69] M. Colpi, S.L. Shapiro, I. Wasserman, Phys. Rev. Lett. 57, 2485 (1986)

[70] J.J. van der Bij, M. Gleiser, Phys. Lett. B 194, 482 (1987)

[71] M. Gleiser, Phys. Rev. D 38, 2376 (1988)

[72] M. Gleiser, R. Watkins, Nucl. Phys. B 319, 733 (1989)

[73] E. Seidel, W.M. Suen, Phys. Rev. D 42, 384 (1990)

[74] F.V. Kusmartsev, E.W. Mielke, F.E. Schunck, Phys. Lett. A 157, 465 (1991)

[75] F.V. Kusmartsev, E.W. Mielke, F.E. Schunck, Phys. Rev. D 43, 3895 (1991)

[76] T.D. Lee, Y. Pang, Phys. Rep. 221, 251 (1992)

[77] P. Jetzer, Phys. Rep. 220, 163 (1992)

[78] E. Seidel, W.M. Suen, Phys. Rev. Lett. 72, 2516 (1994)

[79] J. Balakrishna, E. Seidel, W.M. Suen, Phys. Rev. D 58, 104004 (1998)

[80] F.E. Schunck, A.R. Liddle, Black Holes: Theory and Observation, Proceedings of the 179th W. E. Heraeus Seminar. Edited by Friedrich W. Hehl, Claus Kiefer, and Ralph J. K. Metzler. Springer (1998), P. 285

[81] E.W. Mielke, F.E. Schunck, Nuc. Phys. B 564, 185 (2000)

[82] D.F. Torres, S. Capozziello, G. Lambiase, Phys. Rev. D 62, $104012(2000)$

[83] X.Z. Wang, Phys. Rev. D 64, 124009 (2001)

[84] F.E. Schunck, E.W. Mielke, Class. Quantum Grav. 20, R301 (2003)

[85] F.S. Guzmán, Phys. Rev. D 73, 021501 (2006)

[86] P.H. Chavanis, T. Harko, Phys. Rev. D 86, 064011 (2012)

[87] J.R. Oppenheimer, G.M. Volkoff, Phys. Rev. 55, 374 (1939)

[88] J. M. Lattimer and M. Prakash, in From Nuclei to Stars, Ed: S. Lee (Singapore, World Scientific, 2011), arXiv:1012.3208

[89] P. B. Demorest, T. Pennucci, S. M. Ransom, M. S. E. Roberts and J. W. T. Hessels, Nature 467, 1081 (2010)

[90] O. Barziv, L. Karper, M. H. van Kerkwijk, J. H. Telging, and J. van Paradijs, Astron. Astrophys.377, 925 (2001)

[91] H. Quaintrell, A. J. Norton, T. D. C. Ash, P. Roche, B. Willems, T. R. Bedding, I. K. Baldry, and R. P. Fender, Astron. Astrophys. 401, 303 (2003)
[92] M. H. van Kerkwijk, R. Breton, and S. R. Kulkarni, Astrophys. J. 728, 95 (2011)

[93] G. Dvali, C. Gomez, Fortschr. Phys. 61, 742 (2013)

[94] R. Casadio, A. Orlandi, J. High Energy Phys. 8, 25 (2013)

[95] R.F. Tooper, Astrophys. J. 140, 434 (1964)

[96] R.F. Tooper, Astrophys. J. 142, 1541 (1965)

[97] Ya. B. Zel'dovich, Soviet Phys. JETP 14, 1143 (1962)

[98] T. Harko, Mon. Not. R. Astron. Soc. 413, 3095 (2011)

[99] Ya. B. Zel'dovich, Mon. Not. R. Astron. Soc. 160, 1 (1972)

[100] S. Chandrasekhar, An Introduction to the Study of Stellar Structure (Dover, 1958)

[101] N. K. Glendenning, Compact Stars, Nuclear Physics, Particle Physics and General Relativity (Springer, New York, 2000)

[102] P.H. Chavanis, Astron. Astrophys. 381, 709 (2002)

[103] P.H. Chavanis, Astron. Astrophys. 483, 673 (2008)

[104] C.W. Misner, H.S. Zapolsky, Phys. Rev. Lett. 12, 635 (1964)

[105] D.W. Meltzer, K.S. Thorne, Astrophys. J. 145, 514 (1966)

[106] P.H. Chavanis, Astron. Astrophys. 381, 340 (2002)

[107] H. Poincaré, Acta Math. 7, 259 (1885)

[108] J. Katz, Mon. Not. R. Astron. Soc. 183, 765 (1978)

[109] P.H. Chavanis, Int. J. Mod. Phys. B 20, 3113 (2006)

[110] S.L. Shapiro, S.A. Teukolsky, Black holes, white dwarfs and neutron stars (Wiley, New York, 1983)

[111] P.H. Chavanis, Astron. Astrophys. 451, 109 (2006)

[112] H. A. Buchdahl, Phys. Rev. 116, 1027 (1959)

[113] A. Mukherjee, S. Shah, S. Bose, arXiv:1409.6490

[114] F. Ozel, G. Baym, T. Guver, Phys. Rev. D 82, 101301 (2010)

[115] F. Ozel, A. Gould, T. Guver, Astrophys. J. 748, 5 (2012)

[116] T. Guver, P. Wroblewski, L. Camarota, F. Ozel, Astrophys. J. 719, 1807 (2010)

[117] S. van Straaten, E.C. ford, M. van der Klis, M. Mendez, P. Kaaret, astro-ph/0001480

[118] P.H. Chavanis, Eur. Phys. J. Plus 129, 38 (2014)

[119] P.H. Chavanis, Eur. Phys. J. Plus 129, 222 (2014)

[120] P.H. Chavanis, arXiv:1208.1185

[121] P.H. Chavanis, arXiv:1309.5784

[122] J. Binney, S. Tremaine, Galactic Dynamics (Princeton, NJ: Princeton University Press, 1987)

[123] P.H. Chavanis, preprint

[124] S. Weinberg, Gravitation and Cosmology (John Wiley, 2002)

[125] H. Bondi, Proc. Roy. Soc. (London) A282, 303 (1964)

[126] B.K. Harrison, K.S. Thorne, M. Wakano, J.A. Wheeler, Gravitation Theory and Gravitational Collapse (Chicago, University of Chicago Press, 1965)

[127] S. Chandrasekhar, Astrophys. J. 74, 81 (1931)

[128] S. Chandrasekhar, Monthly Not. Roy. Astron. Soc. 74, 81 (1931) 